\title{
On backward problem for fractional spherically symmetric diffusion equation with observation data of nonlocal type
}

\author{
Le Dinh Long ${ }^{1}$, Ho Thi Kim Van ${ }^{1}$, Ho Duy Binh ${ }^{1}$ and Reza Saadati ${ }^{*}$ (1)
}

"Correspondence: rsaadati@eml.cc ${ }^{2}$ School of Mathematics, Iran University of Science and Technology, Narmak, Tehran, Iran Full list of author information is available at the end of the article

\section{Springer}

\begin{abstract}
The main target of this paper is to study a problem of recovering a spherically symmetric domain with fractional derivative from observed data of nonlocal type. This problem can be established as a new boundary value problem where a Cauchy condition is replaced with a prescribed time average of the solution. In this work, we set some of the results above existence and regularity of the mild solutions of the proposed problem in some suitable space. Next, we also show the ill-posedness of our problem in the sense of Hadamard. The regularized solution is given by the fractional Tikhonov method and convergence rate between the regularized solution and the exact solution under a priori parameter choice rule and under a posteriori parameter choice rule.
\end{abstract}

MSC: 35K05; 35K99; 47J06; 47H10x

Keywords: Backward in time; Fractional spherically symmetric diffusion equation; Fractional Tikhonov method; IIl-posed problem; Convergence estimates;

Regularization

\section{Introduction}

In recent decades, the study of noninteger diffusion equations has received great attention from mathematicians around the world. These models have many applications in various types of research fields, for example, thermal diffusion in fractal domains [1] and protein dynamics [2], finance [3], systems biology [4], physics [5] and medicine [6], and besides, there are also some references as follows [7-11], and [12]. In this work, we consider the following problem:

$$
\begin{cases}D_{t}^{\beta} u(r, t)=\frac{\partial^{2} u}{\partial r^{2}}+\frac{2}{r} \frac{\partial u}{\partial r}+G(r, t), & 0 \leq r \leq R, 0 \leq t \leq T \\ u(R, t)=u(0, t)=0, & 0 \leq t \leq T, \\ \lim _{r \rightarrow 0} u(r, t) \text { bounded, } & 0 \leq t \leq T, \\ \xi_{1} u(r, T)+\xi_{2} \int_{0}^{T} u(r, t) d t=f(r) . & \end{cases}
$$

(c) The Author(s) 2021. This article is licensed under a Creative Commons Attribution 4.0 International License, which permits use sharing, adaptation, distribution and reproduction in any medium or format, as long as you give appropriate credit to the original author(s) and the source, provide a link to the Creative Commons licence, and indicate if changes were made. The images or other third party material in this article are included in the article's Creative Commons licence, unless indicated otherwise in a credit line to the material. If material is not included in the article's Creative Commons licence and your intended use is not permitted by statutory regulation or exceeds the permitted use, you will need to obtain permission directly from the copyright holder. To view a copy of this licence, visit http://creativecommons.org/licenses/by/4.0/ 
Here Caputo fractional derivative $D_{t}^{\beta}$ is defined as follows:

$$
D_{t}^{\beta} u(r, t)=\frac{1}{\Gamma(1-\beta)} \int_{0}^{t} \frac{u_{s}(r, s)}{(t-s)^{\beta}} d s, \quad 0<\beta<1,
$$

and the source function $G(r, t) \in L^{\infty}\left([0, R] ; r^{2}\right)$, the final data $f(r) \in L^{2}\left([0, R], r^{2}\right)$ are given. Note that when the fractional order $\beta$ is equal to 1 , the fractional derivative $D_{t}^{\beta} u(r, t)$ is equal to the first-order derivative $\frac{d u}{d t}$ (see in [13]), and thus problem (1.1) reproduces the classical diffusion problem. In practice, the input data $(f, G)$ is noisy by the observed data $f^{\varepsilon}, G^{\varepsilon}$ which satisfy

$$
\left\|f^{\epsilon}-f\right\|_{L^{2}\left([0, R] ; r^{2}\right)}+\left\|G^{\epsilon}-G\right\|_{L^{\infty}\left(0, T ; L^{2}\left([0, R] ; r^{2}\right)\right)} \leq \epsilon .
$$

Our problem is called inverse problem and its solution is not stable. This property is called ill-posed in the sense of Hadamard. In other words, easier to understand, if $\epsilon$ is small, it will lead to large errors for the corresponding solution if using an unapproximate model for observed data $f^{\varepsilon}, G^{\varepsilon}$. The question mentioned in this paper is: Find an approximation method for the solution of the problem with noisy input data $f^{\varepsilon}, G^{\varepsilon}$.

Before discussing the main results, we would like to outline a few previous papers that mentioned problem (1.1).

- If $\beta=1, \xi_{2}=0, \xi_{1}=1$, and $G=0$, then the last condition in (1.1) becomes the final condition

$$
u(r, T)=f(r)
$$

In such a case, problem (1.1) is called backward problem. Then the authors [14] used a modified Tikhonov regularization method for solving problem. In [15], the authors used a spectral method for regularizing the problem.

- If $\beta=1, \xi_{2}=0, \xi_{1}=1$, and $G(r, t)=\varphi(t) f(r)$, WeiCheng et al. [16] applied a spectral method to approximate the backward problem and obtained Hölder type estimate with a suitable choice of regularization parameter.

- If $\beta \neq 1$ and $\xi_{2}=0, \xi_{1}=1$ and $G(r, t)=0$, then the authors [17] proposed the quasi-boundary regularization method to solve problem (1.1). They showed convergence estimates between the regularization solution and the exact solution presented under the a priori and a posteriori regularization parameter choice rules.

- If $\beta \neq 1$ and $\xi_{2}=0, \xi_{1}=1$ and $G(r, t)=f(r)$, Yang et al. [18] investigated problem (1.1) and provided the estimate of Hölder type.

Let us mention some interesting papers with many various methods for the case $G(r, t) \neq$ 0 , for example, the truncation method [19], iterated fractional Tikhonov regularization method [20], and the references therein. Besides, regarding other regularization methods and applications, readers can view the following references: [21-28].

Our novel point in this paper is to replace the final condition (1.4) with the nonlocal condition $\xi_{1} u(r, T)+\xi_{2} \int_{0}^{T} u(r, t) d t=f(r)$ as introduced in the last condition of our problem. This condition is proposed in the paper by Dokuchaev [29]. Very recently, Tuan and coauthors used this condition to solve some nonlocal problem, for example [30-32], and [33]. Motivated by this above reason, in this paper, we apply the fractional Tikhonov method to 
solve problem (1.1). To the best of authors' knowledge, there are not any results concerning problem (1.1). Our paper investigates problem (1.1), and the main results of this work are as follows:

- We give the stability and the regularity of the mild solution.

- We show the ill-posedness and the conditional stability of solution in $L^{2}\left([0, R] ; r^{2}\right)$.

- We propose a regularized method and prove the convergence rate under a priori parameter choice rule and a posteriori parameter choice rule.

Let us say that in an analytical sense, our problem seems to be more complicated than the models studied before.

This paper is organized as follows. Section 2 gives some preliminaries that are needed throughout the paper. In Sect. 3, we show the sought solution of problem (1.1), and an example describes the ill-posedness of the problem. In Sect. 4, we study the fractional Tikhonov method to solve problem (1.1) and show the convergence rate under a priori parameter choice rule and a posteriori parameter choice rule. Finally, we add the conclusion for this paper.

\section{Preliminaries}

In this paper, we denote by $L^{2}\left([0, R] ; r^{2}\right)$ the Hilbert space of Lebesgue measurable functions $u(r, t)$ with weight $r^{2}$ on $[0, R]$

$$
\langle u, v\rangle=\int_{0}^{R} r^{2} u(r) v(r) d r
$$

with the scalar product $\|u\|_{L^{2}\left([0, R], r^{2}\right)}=\left(\int_{0}^{R} r^{2}|u(r)|^{2} d r\right)^{\frac{1}{2}}$, and the $L^{\infty}(0, T ; Y)$ consists of all measurable functions $u:[0, T] \rightarrow Y$ with

$$
\|u\|_{L^{\infty}(0, T ; Y)}:=\operatorname{ess} \sup _{0 \leq t \leq T}\|u(t)\|_{Y}
$$

where $Y$ is a real Banach space with the norm $\|\cdot\|_{Y}$. We define the space

$$
\mathcal{H}^{s}\left([0, R] ; r^{2}\right)=\left\{v \in L^{2}\left([0, R], r^{2}\right): \sum_{j=1}^{+\infty}\left(j^{2}\right)^{2 s}\left|\left\langle\nu, \psi_{j}\right\rangle\right|^{2}<+\infty\right\},
$$

then $\mathcal{H}^{s}\left([0, R] ; r^{2}\right)$ is a Hilbert space with the norm

$$
\|v\|_{\mathcal{H}^{s}\left([0, R] ; r^{2}\right)}=\left(\sum_{j=1}^{+\infty}\left(j^{2}\right)^{2 s}\left|\left\langle\nu, \psi_{j}\right\rangle\right|^{2}\right)^{\frac{1}{2}}
$$

For any constant $\alpha>0$ and $\beta \in \mathbb{R}$, the Mittag-Leffler function is defined as follows:

$$
\mathrm{E}_{\alpha, \beta}(z)=\sum_{k=0}^{\infty} \frac{z^{k}}{\Gamma(\alpha k+\beta)}, \quad z \in \mathbb{C},
$$

where $\alpha>0$ and $\beta \in \mathbb{R}$ are arbitrary constants. 
Lemma 2.1 ([18]) Assuming that $0<\beta_{0}<\beta_{1}<1$, then there exist constants $\bar{D}_{1}$ and $\bar{D}_{2}$ depending only on $\beta, \beta_{1}$ such that for all $\beta \in\left[\beta_{0}, \beta_{1}\right]$ there hold

$$
\frac{\bar{D}_{1}}{\Gamma(1-\beta)} \frac{1}{1-z} \leq E_{\beta, 1}(z) \leq \frac{\bar{D}_{2}}{\Gamma(1-\beta)} \frac{1}{1-z}, \quad z \geq 0
$$

Lemma 2.2 ([19]) For $\lambda_{j} \geq \lambda_{1}>0, \lambda_{j}$ is the eigenvalues satisfying $\lambda_{1}<\lambda_{2}<\cdots<\lambda_{j} \rightarrow$ $\infty$ as $j \rightarrow \infty$, then there exist constants $\bar{D}_{3}$ and $\bar{D}_{4}$ depending only on $\beta, T, \lambda_{1}$ such that

$$
\frac{\bar{D}_{3} R^{2}}{j^{2} \pi^{2}} \leq E_{\beta, \beta}\left(-\left(\frac{j \pi}{R}\right)^{2} T^{\beta}\right) \leq \frac{\bar{D}_{4} R^{2}}{j^{2} \pi^{2}}
$$

Proof This proof can be found in [20].

Lemma 2.3 Let $G \in L^{\infty}\left(0, T ; L^{2}\left([0, T], r^{2}\right)\right)$, then the exists a positive constant $\bar{D}_{5}$ such that

$$
\begin{aligned}
& \sum_{j=1}^{+\infty}\left|\int_{0}^{t}(t-s)^{\beta-1} E_{\beta, \beta}\left(-\left(\frac{j \pi}{R}\right)^{2}(t-s)^{\beta}\right)\left\langle G(\cdot, s), \psi_{j}\right\rangle d s\right|^{2} \\
& \quad \leq \bar{D}_{5}\|G\|_{L^{\infty}\left(0, T ; L^{2}\left([0, T], r^{2}\right)\right)}^{2} .
\end{aligned}
$$

Proof This lemma provision can be found in [20].

Lemma 2.4 For any $j \geq 1$, we have the following estimate:

$$
\frac{\underline{E}}{j^{2}} \leq \xi_{1} E_{\beta, 1}\left(-\left(\frac{j \pi}{R}\right)^{2} T^{\beta}\right)+\xi_{2} \int_{0}^{T} E_{\beta, 1}\left(-\left(\frac{j \pi}{R}\right)^{2} t^{\beta}\right) d t \leq \frac{\bar{E}}{j^{2}}
$$

in which $\underline{E}=\left(\frac{\bar{D}_{3} R^{2}}{\pi^{2}}+\frac{\xi_{2} \bar{D}_{3} R^{2}}{\pi^{2}} \frac{T}{\frac{R^{2}}{\pi^{2}}+T^{\beta}}\right), \bar{E}=\left(\frac{\xi_{1} \bar{D}_{4} R^{2}}{\pi^{2}}+\frac{\xi_{2} \bar{D}_{4} R^{2}}{\pi^{2}} \frac{T^{1-\beta}}{1-\beta}\right)$.

Proof From Lemma 2.2, we need to show that

$$
\xi_{2} \int_{0}^{T} E_{\beta, 1}\left(-\left(\frac{j \pi}{R}\right)^{2} t^{\beta}\right) d t \leq \xi_{2} \bar{D}_{4} \int_{0}^{T} \frac{1}{1+\left(\frac{j \pi}{R}\right)^{2} t^{\beta}} d t \leq \frac{\xi_{2} \bar{D}_{4} R^{2}}{j^{2} \pi^{2}} \frac{T^{1-\beta}}{1-\beta} .
$$

This leads to

$$
\xi_{1} E_{\beta, 1}\left(-\left(\frac{j \pi}{R}\right)^{2} T^{\beta}\right)+\xi_{2} \int_{0}^{T} E_{\beta, 1}\left(-\left(\frac{j \pi}{R}\right)^{2} t^{\beta}\right) d t \leq \frac{\bar{E}}{j^{2}} .
$$

Next, due to the fact, we also get

$$
\xi_{2} \int_{0}^{T} E_{\beta, 1}\left(-\left(\frac{j \pi}{R}\right)^{2} t^{\beta}\right) d t \geq \frac{\xi_{2} \bar{D}_{3} R^{2}}{j^{2} \pi^{2}} \int_{0}^{T} \frac{d t}{\frac{R^{2}}{\pi^{2}}+T^{\beta}}=\frac{\xi_{2} \bar{D}_{3} R^{2}}{j^{2} \pi^{2}} \frac{T}{\frac{R^{2}}{\pi^{2}}+T^{\beta}},
$$


which implies that

$$
\xi_{1} E_{\beta, 1}\left(-\left(\frac{j \pi}{R}\right)^{2} T^{\beta}\right)+\xi_{2} \int_{0}^{T} E_{\beta, 1}\left(-\left(\frac{j \pi}{R}\right)^{2} t^{\beta}\right) d t \geq \frac{E}{j^{2}} .
$$

In this section, we need the solution of the direct problem of (1.1)

$$
\begin{cases}D_{t}^{\beta} u(r, t)=\frac{\partial^{2} u}{\partial r^{2}}+\frac{1}{r} \frac{\partial u}{\partial r}+G(r, t), & 0 \leq r \leq R, 0 \leq t \leq T, \\ u(r, 0)=\ell(r), & 0 \leq r \leq R, \\ \lim _{r \rightarrow 0} u(r, t) \text { bounded, } \quad u(R, t)=0, & 0 \leq t \leq T .\end{cases}
$$

From [20], by using the Fourier expansion, we know that

$$
\begin{aligned}
u_{j}(t)= & E_{\beta, 1}\left(-\left(\frac{j \pi}{R}\right)^{2} t^{\beta}\right)\left\langle\ell, \psi_{j}\right\rangle \\
& +\int_{0}^{t}(t-s)^{\beta-1} E_{\beta, \beta}\left(-\left(\frac{j \pi}{R}\right)^{2}(t-s)^{\beta}\right)\left\langle G(\cdot, s), \psi_{j}\right\rangle d s,
\end{aligned}
$$

where $\psi_{j}(r)=\frac{\sqrt{2} j \pi}{\sqrt{R^{3}}} j_{0}\left(\frac{j \pi r}{R}\right)$, and $j_{0}(y)$ denotes the 0 th order spherical Bessel functions of the first kind. Besides, we know that $\left\{\psi_{j}(r)\right\}_{j=1}^{\infty}$ from an orthonormal basis in $L^{2}\left([0, T], r^{2}\right)$.

\section{The mild solution of problem (1.1)}

Theorem 3.1 Let $f \in L^{2}\left([0, R] ; r^{2}\right)$ and $G \in L^{\infty}\left(0, T ; L^{2}([0, R] ; r)\right)$. Let us further assume that

$$
\sum_{j=1}^{+\infty} \frac{\left\langle f, \psi_{j}\right\rangle-\xi_{1} \int_{0}^{T}(T-s)^{\beta-1} E_{\beta, \beta}\left(-\left(\frac{j \pi}{R}\right)^{2}(T-s)^{\beta}\right)\left\langle G(\cdot, s), \psi_{j}\right\rangle d s}{\xi_{1} E_{\beta, 1}\left(-\left(\frac{j \pi}{R}\right)^{2} T^{\beta}\right)+\xi_{2} \int_{0}^{T} E_{\beta, 1}\left(-\left(\frac{j \pi}{R}\right)^{2} t^{\beta}\right) d t}<\infty .
$$

Then problem (1.1) has a unique solution $u$ given as follows:

$$
\begin{aligned}
u(r, t) & \\
= & \sum_{j=1}^{+\infty} \frac{E_{\beta, 1}\left(-\left(\frac{j \pi}{R}\right)^{2} t^{\beta}\right)\left\langle f, \psi_{j}\right\rangle}{\left(\xi_{1} E_{\beta, 1}\left(-\left(\frac{j \pi}{R}\right)^{2} T^{\beta}\right)+\xi_{2} \int_{0}^{T} E_{\beta, 1}\left(-\left(\frac{j \pi}{R}\right)^{2} t^{\beta}\right) d t\right)} \psi_{j}(r) \\
& -\sum_{j=1}^{+\infty} \frac{\xi_{1} E_{\beta, 1}\left(-\left(\frac{j \pi}{R}\right)^{2} t^{\beta}\right) \int_{0}^{T}(T-s)^{\beta-1} E_{\beta, \beta}\left(-\left(\frac{j \pi}{R}\right)^{2}(T-s)^{\beta}\right)\left\langle G(\cdot, s), \psi_{j}\right\rangle d s}{\left(\xi_{1} E_{\beta, 1}\left(-\left(\frac{j \pi}{R}\right)^{2} T^{\beta}\right)+\xi_{2} \int_{0}^{T} E_{\beta, 1}\left(-\left(\frac{j \pi}{R}\right)^{2} t^{\beta}\right) d t\right)} \psi_{j}(r) \\
& -\sum_{j=1}^{+\infty} \frac{\xi_{2} E_{\beta, 1}\left(-\left(\frac{j \pi}{R}\right)^{2} t^{\beta}\right) \int_{0}^{T}\left(\int_{0}^{t}(t-s)^{\beta-1} E_{\beta, \beta}\left(-\left(\frac{j \pi}{R}\right)^{2}(t-s)^{\beta}\right)\left\langle G(\cdot, s), \psi_{j}\right\rangle\right) d t}{\left(\xi_{1} E_{\beta, 1}\left(-\left(\frac{j \pi}{R}\right)^{2} T^{\beta}\right)+\xi_{2} \int_{0}^{T} E_{\beta, 1}\left(-\left(\frac{j \pi}{R}\right)^{2} t^{\beta}\right) d t\right)} \psi_{j}(r) \\
& \left.+\sum_{j=1}^{+\infty} \int_{0}^{t}(t-s)^{\beta-1} E_{\beta, \beta}\left(-\left(\frac{j \pi}{R}\right)^{2}(t-s)^{\beta}\right)\left\langle G(\cdot, s), \psi_{j}\right\rangle d s\right) \psi_{j}(r) .
\end{aligned}
$$

Then we get the following regularity:

$$
\|u(\cdot, t)\|_{L^{2}\left([0, R] ; r^{2}\right)} \leq 2 \mathcal{M}\|f\|_{L^{2}\left([0, R] ; r^{2}\right)}+2 \bar{M}\|G\|_{L^{\infty}\left(0, T ; L^{2}\left([0, R] ; r^{2}\right)\right)} .
$$


Here $\mathcal{M}, \bar{M}$ are defined later. Let $f \in \mathcal{H}^{s}\left([0, R] ; r^{2}\right)$ and $G \in L^{2}\left(0, T ; \mathcal{H}\left([0, R] ; r^{2}\right)\right) \cap$ $L^{\infty}\left(0, T ; \mathcal{H}^{s-\gamma}\left([0, R] ; r^{2}\right)\right)$ for $s>\gamma+1$, then $u \in L^{p}\left(0, T ; \mathcal{H}^{s}\left([0, R] ; r^{2}\right)\right)$ and the regularity result holds

$$
\begin{aligned}
\|u(\cdot, t)\|_{L^{p}\left(0, T ; \mathcal{H}^{s}\left([0, R] ; r^{2}\right)\right)} \lesssim & \|f\|_{\mathcal{H}^{s}\left([0, R] ; r^{2}\right)}+\|G\|_{L^{\infty}\left(0, T ; \mathcal{H}^{s-\gamma}\left([0, R] ; r^{2}\right)\right)} \\
& +\|G\|_{L^{2}\left(0, T ; \mathcal{H}^{s-1-\gamma}\left([0, R] ; r^{2}\right)\right)} .
\end{aligned}
$$

Proof From (2.15) and using the nonlocal condition in problem (1.1), we obtain that

$$
\begin{aligned}
\xi_{1} u_{j}(T)= & \xi_{1}\left\langle\ell, \psi_{j}\right\rangle E_{\beta, 1}\left(-\left(\frac{j \pi}{R}\right)^{2} T^{\beta}\right) \\
& +\xi_{1} \int_{0}^{T}(T-s)^{\beta-1} E_{\beta, \beta}\left(-\left(\frac{j \pi}{R}\right)^{2}(T-s)^{\beta}\right)\left\langle G(\cdot, s), \psi_{j}\right\rangle d s .
\end{aligned}
$$

By integrating both sides from 0 to $T$ for equation (2.15), we immediately have the following equality:

$$
\begin{aligned}
\xi_{2} \int_{0}^{T} u_{j}(t) d t= & \xi_{2}\left\langle\ell, \psi_{j}\right\rangle \int_{0}^{T} E_{\beta, 1}\left(-\left(\frac{j \pi}{R}\right)^{2} t^{\beta}\right) d t \\
& +\xi_{2} \int_{0}^{T}\left(\int_{0}^{t}(t-s)^{\beta-1} E_{\beta, \beta}\left(-\left(\frac{j \pi}{R}\right)^{2}(t-s)^{\beta}\right)\left\langle G(\cdot, s), \psi_{j}\right\rangle\right) d t .
\end{aligned}
$$

From two observations (3.5) and (3.6), we get the following equality:

$$
\begin{aligned}
\xi_{1} u_{j}(T) & +\xi_{2} \int_{0}^{T} u_{j}(t) d t \\
= & \left\langle\ell, \psi_{j}\right\rangle\left(\xi_{1} E_{\beta, 1}\left(-\left(\frac{j \pi}{R}\right)^{2} T^{\beta}\right)+\xi_{2} \int_{0}^{T} E_{\beta, 1}\left(-\left(\frac{j \pi}{R}\right)^{2} t^{\beta}\right)\right) \\
& +\xi_{1} \int_{0}^{T}(T-s)^{\beta-1} E_{\beta, \beta}\left(-\left(\frac{j \pi}{R}\right)^{2}(T-s)^{\beta}\right)\left\langle G(\cdot, s), \psi_{j}\right\rangle d s \\
& +\xi_{2} \int_{0}^{T}\left(\int_{0}^{t}(t-s)^{\beta-1} E_{\beta, \beta}\left(-\left(\frac{j \pi}{R}\right)^{2}(t-s)^{\beta}\right)\left\langle G(\cdot, s), \psi_{j}\right\rangle\right) d t .
\end{aligned}
$$

Our next aim is to express the formula of the function $\ell$ in terms of two input data $f$ and $G$. In view of the nonlocal condition as in the last condition of problem (1.1)

$$
\xi_{1} u_{j}(T)+\xi_{2} \int_{0}^{T} u_{j}(t) d t=f(r)
$$

we find the following identity for the Fourier coefficient of the function $\ell$ :

$$
\begin{aligned}
\left\langle\ell, \psi_{j}\right\rangle= & \frac{\left\langle f, \psi_{j}\right\rangle}{\xi_{1} E_{\beta, 1}\left(-\left(\frac{j \pi}{R}\right)^{2} T^{\beta}\right)+\xi_{2} \int_{0}^{T} E_{\beta, 1}\left(-\left(\frac{j \pi}{R}\right)^{2} t^{\beta}\right) d t} \\
& -\frac{\xi_{1} \int_{0}^{T}(T-s)^{\beta-1} E_{\beta, \beta}\left(-\left(\frac{j \pi}{R}\right)^{2}(T-s)^{\beta}\right)\left\langle G(\cdot, s), \psi_{j}\right\rangle d s}{\xi_{1} E_{\beta, 1}\left(-\left(\frac{j \pi}{R}\right)^{2} T^{\beta}\right)+\xi_{2} \int_{0}^{T} E_{\beta, 1}\left(-\left(\frac{j \pi}{R}\right)^{2} t^{\beta}\right) d t} \\
& -\frac{\xi_{2} \int_{0}^{T}\left(\int_{0}^{t}(t-s)^{\beta-1} E_{\beta, \beta}\left(-\left(\frac{j \pi}{R}\right)^{2}(t-s)^{\beta}\right)\left\langle G(\cdot, s), \psi_{j}\right\rangle d s\right) d t}{\xi_{1} E_{\beta, 1}\left(-\left(\frac{j \pi}{R}\right)^{2} T^{\beta}\right)+\xi_{2} \int_{0}^{T} E_{\beta, 1}\left(-\left(\frac{j \pi}{R}\right)^{2} t^{\beta}\right) d t} .
\end{aligned}
$$


Therefore, by taking Fourier series for the term $u_{j}(t)$, the formula of the mild solution to problem (1.1) can be given by

$$
\begin{aligned}
u(r, t)= & \underbrace{\sum_{j=1}^{+\infty} \frac{E_{\beta, 1}\left(-\left(\frac{j \pi}{R}\right)^{2} t^{\beta}\right)\left\langle f, \psi_{j}\right\rangle}{\xi_{1} E_{\beta, 1}\left(-\left(\frac{j \pi}{R}\right)^{2} T^{\beta}\right)+\xi_{2} \int_{0}^{T} E_{\beta, 1}\left(-\left(\frac{j \pi}{R}\right)^{2} t^{\beta}\right) d t} \psi_{j}(r)}_{:=\mathcal{A}_{1}(r, t)} \\
& -\underbrace{\sum_{j=1}^{+\infty} \frac{\xi_{1} E_{\beta, 1}\left(-\left(\frac{j \pi}{R}\right)^{2} t^{\beta}\right) \int_{0}^{T}(T-s)^{\beta-1} E_{\beta, \beta}\left(-\left(\frac{j \pi}{R}\right)^{2}(T-s)^{\beta}\right)\left\langle G(\cdot, s), \psi_{j}\right\rangle d s}{\xi_{1} E_{\beta, 1}\left(-\left(\frac{j \pi}{R}\right)^{2} T^{\beta}\right)+\xi_{2} \int_{0}^{T} E_{\beta, 1}\left(-\left(\frac{j \pi}{R}\right)^{2} t^{\beta}\right) d t} \psi_{j}(r)}_{:=\mathcal{A}_{2}(r, t)} \\
& -\underbrace{\sum_{j=1}^{+\infty} \frac{\xi_{2} E_{\beta, 1}\left(-\left(\frac{j \pi}{R}\right)^{2} t^{\beta}\right) \int_{0}^{T}\left(\int_{0}^{t}(t-s)^{\beta-1} E_{\beta, \beta}\left(-\left(\frac{j \pi}{R}\right)^{2}(t-s)^{\beta}\right)\left\langle G(\cdot, s), \psi_{j}\right\rangle\right) d t}{\xi_{1} E_{\beta, 1}\left(-\left(\frac{j \pi}{R}\right)^{2} T^{\beta}\right)+\xi_{2} \int_{0}^{T} E_{\beta, 1}\left(-\left(\frac{j \pi}{R}\right)^{2} t^{\beta}\right) d t}}_{:=\mathcal{A}_{3}(r, t)} \psi_{j}(r) \\
& +\underbrace{\left.\sum_{j=1}^{+\infty} \int_{0}^{t}(t-s)^{\beta-1} E_{\beta, \beta}\left(-\left(\frac{j \pi}{R}\right)^{2}(t-s)^{\beta}\right)\left\langle G(\cdot, s), \psi_{j}\right\rangle d s\right) \psi_{j}(r)}_{:=\mathcal{A}_{4}(r, t)} .
\end{aligned}
$$

Using the inequality $(a+b+c+d)^{2} \leq 4\left(a^{2}+b^{2}+c^{2}+d^{2}\right)$, for $a, b, c, d \geq 0$, we have

$$
\begin{aligned}
\|u\|_{L^{2}\left([0, R] ; r^{2}\right)}^{2} \leq & 4\left\|\mathcal{A}_{1}(\cdot, t)\right\|_{L^{2}\left([0, R] ; r^{2}\right)}^{2}+4\left\|\mathcal{A}_{2}(\cdot, t)\right\|_{L^{2}\left([0, R] ; r^{2}\right)}^{2} \\
& +4\left\|\mathcal{A}_{3}(\cdot, t)\right\|_{L^{2}\left([0, R] ; r^{2}\right)}^{2}+4\left\|\mathcal{A}_{4}(\cdot, t)\right\|_{L^{2}\left([0, R] ; r^{2}\right)}^{2} .
\end{aligned}
$$

Now, we give the regularity result of a mild solution. First of all, from Lemma 2.4, it gives

$$
\begin{aligned}
& \sup _{j \geq 1}\left|\frac{E_{\beta, 1}\left(-\left(\frac{j \pi}{R}\right)^{2} t^{\beta}\right)}{\xi_{1} E_{\beta, 1}\left(-\left(\frac{j \pi}{R}\right)^{2} T^{\beta}\right)+\xi_{2} \int_{0}^{T} E_{\beta, 1}\left(-\left(\frac{j \pi}{R}\right)^{2} t^{\beta}\right) d t}\right|^{2} \\
& \quad \leq \frac{j^{4}}{\underline{E}^{2}}\left(\frac{\bar{D}_{2}}{\Gamma(1-\beta)} \frac{1}{1+\left(\frac{j \pi}{R}\right)^{2} t^{\beta}}\right)^{2} \leq\left(\frac{\bar{D}_{2}}{\underline{E} \Gamma(1-\beta)}\right)^{2}\left|\frac{1}{\left(\frac{\pi}{R}\right)^{2} t^{\beta}}\right|^{2} \\
& \quad \leq\left(\frac{\bar{D}_{2}}{\underline{E} \Gamma(1-\beta)}\right)^{2}\left(\frac{R^{2}}{\pi^{2} t^{\beta}}\right)^{2} .
\end{aligned}
$$

From now on, in short, we denote $\mathcal{M}^{2}\left(\beta, \underline{E}, R, \pi, t, \bar{D}_{2}\right)=\left(\frac{\bar{D}_{2}}{E \Gamma(1-\beta)}\right)^{2}\left(\frac{R^{2}}{\pi^{2} t^{\beta}}\right)^{2}$. Next, let us evaluate for $\left\|\mathcal{A}_{i}(\cdot, t)\right\|_{L^{2}\left([0, T] ; r^{2}\right)^{2}}^{2}, i=\overline{1,4}$, one by one.

Step 1: Estimate of $\left\|\mathcal{A}_{1}(\cdot, t)\right\|_{L^{2}\left([0, T] ; r^{2}\right)^{2}}^{2}$. By using the estimation for (3.11), we have

$$
\begin{aligned}
\left\|\mathcal{A}_{1}(\cdot, t)\right\|_{L^{2}\left([0, R] ; r^{2}\right)}^{2} & \leq \sum_{j=1}^{+\infty}\left[\frac{E_{\beta, 1}\left(-\left(\frac{j \pi}{R}\right)^{2} t^{\beta}\right)\left\langle f, \psi_{j}\right\rangle}{\xi_{1} E_{\beta, 1}\left(-\left(\frac{j \pi}{R}\right)^{2} T^{\beta}\right)+\xi_{2} \int_{0}^{T} E_{\beta, 1}\left(-\left(\frac{j \pi}{R}\right)^{2} t^{\beta}\right) d t}\right]^{2} \\
& \leq \mathcal{M}^{2} \sum_{j=1}^{+\infty}|\langle f, \psi\rangle|^{2} \leq \mathcal{M}^{2}\|f\|_{L^{2}\left([0, T] ; r^{2}\right)}^{2}
\end{aligned}
$$


Step 2: Applying Lemma 2.3 and the estimation of $(3.11),\left\|\mathcal{A}_{2}(\cdot, t)\right\|_{L^{2}\left([0, T] ; r^{2}\right)}^{2}$ can be bounded as follows:

$$
\begin{aligned}
& \left\|\mathcal{A}_{2}(\cdot, t)\right\|_{L^{2}\left([0, R] ; r^{2}\right)}^{2} \\
& \leq \sum_{j=1}^{+\infty}\left[\frac{\xi_{1} E_{\beta, 1}\left(-\left(\frac{j \pi}{R}\right)^{2} t^{\beta}\right) \int_{0}^{T}(T-s)^{\beta-1} E_{\beta, \beta}\left(-\left(\frac{j \pi}{R}\right)^{2}(T-s)^{\beta}\right)\left\langle G(\cdot, s), \psi_{j}\right\rangle d s}{\xi_{1} E_{\beta, 1}\left(-\left(\frac{j \pi}{R}\right)^{2} T^{\beta}\right)+\xi_{2} \int_{0}^{T} E_{\beta, 1}\left(-\left(\frac{j \pi}{R}\right)^{2} t^{\beta}\right) d t}\right]^{2} \\
& \quad \leq \mathcal{M}^{2} \sum_{j=1}^{+\infty}\left[\int_{0}^{T}(T-s)^{\beta-1} E_{\beta, \beta}\left(-\left(\frac{j \pi}{R}\right)^{2}(T-s)^{\beta}\right)\left\langle G(\cdot, s), \psi_{j}\right\rangle d s\right]^{2} \\
& \quad \leq \mathcal{M}^{2} \xi_{1}^{2} \bar{D}_{5}^{2}\|G\|_{L^{2}\left([0, R] ; r^{2}\right)^{.}}^{2}
\end{aligned}
$$

Step 3: Similarly, the estimate of $\left\|\mathcal{A}_{3}(\cdot, t)\right\|_{L^{2}\left([0, T] ; r^{2}\right)}^{2}$ is given by

$$
\begin{aligned}
& \left\|\mathcal{A}_{3}(\cdot, t)\right\|_{L^{2}\left([0, R] ; r^{2}\right)}^{2} \\
& \quad \leq \sum_{j=1}^{+\infty}\left|\frac{\xi_{2} E_{\beta, 1}\left(-\left(\frac{j \pi}{R}\right)^{2} t^{\beta}\right) \int_{0}^{T}\left(\int_{0}^{t}(t-s)^{\beta-1} E_{\beta, \beta}\left(-\left(\frac{j \pi}{R}\right)^{2}(t-s)^{\beta}\right)\left\langle G(\cdot, s), \psi_{j}\right\rangle d s\right) d t}{\xi_{1} E_{\beta, 1}\left(-\left(\frac{j \pi}{R}\right)^{2} T^{\beta}\right)+\xi_{2} \int_{0}^{T} E_{\beta, 1}\left(-\left(\frac{j \pi}{R}\right)^{2} t^{\beta}\right) d t}\right|^{2} \\
& \quad \leq \mathcal{M}^{2} \bar{D}_{5}^{2} \xi_{2}^{2} T^{2}\|G\|_{L^{2}\left([0, R] ; r^{2}\right)}^{2} .
\end{aligned}
$$

Step 4: By using Lemma 2.3, the estimate of $\left\|\mathcal{A}_{4}(\cdot, t)\right\|_{L^{2}\left([0, T] ; r^{2}\right)}^{2}$ is given by

$$
\begin{aligned}
\left\|\mathcal{A}_{4}(\cdot, t)\right\|_{L^{2}\left([0, R] ; r^{2}\right)}^{2} & \leq \sum_{j=1}^{+\infty}\left[\int_{0}^{t}(t-s)^{\beta-1} E_{\beta, \beta}\left(-\left(\frac{j \pi}{R}\right)^{2}(t-s)^{\beta}\right)\left\langle G(\cdot, s), \psi_{j}\right\rangle d s\right]^{2} \\
& \leq \bar{D}_{5}^{2}\|G\|_{L^{2}\left([0, R] ; r^{2}\right)^{.}}^{2}
\end{aligned}
$$

Combining (3.12), (3.13), (3.14), and (3.15), we have the estimate

$$
\|u\|_{L^{2}\left([0, R] ; r^{2}\right)}^{2} \leq 4 \mathcal{M}^{2}\|f\|_{L^{2}\left([0, R] ; r^{2}\right)}^{2}+4\left(\mathcal{M}^{2} \xi_{1}^{2} \bar{D}_{5}^{2}+\mathcal{M}^{2} \xi_{2}^{2} T^{2} \bar{D}_{5}^{2}\right)\|G\|_{L^{2}\left([0, R] ; r^{2}\right)}^{2}
$$

Part B: Similar to part A, let us divide this part into the following steps.

Step 1: Estimate for $\left\|\mathcal{A}_{1}(\cdot, t)\right\|_{\mathcal{H}^{s}\left([0, R] ; r^{2}\right)}$, we have

$$
\begin{aligned}
\left\|\mathcal{A}_{1}(\cdot, t)\right\|_{\mathcal{H}^{s}\left([0, R] ; r^{2}\right)}^{2} & =\sum_{j=1}^{+\infty}\left(j^{2}\right)^{2 s}\left[\frac{E_{\beta, 1}\left(-\left(\frac{j \pi}{R}\right)^{2} t^{\beta}\right)\left\langle f, \psi_{j}\right\rangle}{\xi_{1} E_{\beta, 1}\left(-\left(\frac{j \pi}{R}\right)^{2} T^{\beta}\right)+\xi_{2} \int_{0}^{T} E_{\beta, 1}\left(-\left(\frac{j \pi}{R}\right)^{2} t^{\beta}\right) d t}\right]^{2} \\
& \leq \sum_{j=1}^{+\infty}\left(j^{2}\right)^{2 s} \frac{\bar{D}_{4}^{2} R^{4}}{j^{4} \pi^{4}} t^{-2 \beta} \frac{j^{4}}{\underline{E}^{2}}\left|\left\langle f, \psi_{j}\right\rangle\right|^{2} \\
& \leq\left(\underline{E}^{2}\right)^{-1} \frac{\bar{D}_{4}^{2} R^{4}}{\pi^{4}} t^{-2 \beta} \sum_{j=1}^{+\infty}\left(j^{2}\right)^{2 s}\left|\left\langle f, \psi_{j}\right\rangle\right|^{2} .
\end{aligned}
$$

Denoting $\mathcal{F}_{1}^{2}=\left(\underline{E}^{2}\right)^{-1} \frac{\bar{D}_{4}^{2} R^{4}}{\pi^{4}}$, taking the square root on both sides, we have

$$
\|\mathcal{A}(\cdot, t)\|_{\mathcal{H}^{s}\left([0, R] ; r^{2}\right)} \leq \mathcal{F}_{1} t^{-\beta}\|f\|_{\mathcal{H}^{s}\left([0, R] ; r^{2}\right)} .
$$


Step 2: Estimate for $\left\|\mathcal{A}_{2}(\cdot, t)\right\|_{\mathcal{H}^{s}\left([0, R] ; r^{2}\right)}$, for any $0<\gamma<1$, we receive

$$
\begin{aligned}
E_{\beta, \beta}\left(-\left(\frac{j \pi}{R}\right)^{2}(T-s)^{\beta}\right) & \leq \frac{\bar{D}_{6}}{1+\left(\frac{j \pi}{R}\right)^{2}(T-s)^{\beta}} \\
& \leq \bar{D}_{6}\left(\frac{\pi}{R}\right)^{-2 \gamma}\left(j^{2}\right)^{-\gamma}(T-s)^{-\beta \gamma}
\end{aligned}
$$

Denoting $\mathcal{F}_{2}=\bar{D}_{6}\left(\frac{\pi}{R}\right)^{-2 \gamma}$. Therefore, we can find that

$$
\begin{aligned}
& \left\|\mathcal{A}_{2}(\cdot, t)\right\|_{\mathcal{H}^{s}\left([0, R] ; r^{2}\right)}^{2} \\
& \quad \leq \sum_{j=1}^{+\infty}\left(j^{2}\right)^{2 s}\left[\frac{\xi_{1} E_{\beta, 1}\left(-\left(\frac{j \pi}{R}\right)^{2} t^{\beta}\right) \int_{0}^{T}(T-s)^{\beta-1} E_{\beta, \beta}\left(-\left(\frac{j \pi}{R}\right)^{2}(T-s)^{\beta}\right)\left\langle G(\cdot, s), \psi_{j}\right\rangle d s}{\xi_{1} E_{\beta, 1}\left(-\left(\frac{j \pi}{R}\right)^{2} T^{\beta}\right)+\xi_{2} \int_{0}^{T} E_{\beta, 1}\left(-\left(\frac{j \pi}{R}\right)^{2} t^{\beta}\right) d t}\right]^{2} \\
& \quad \leq \xi_{1}^{2} \sum_{j=1}^{+\infty}\left(j^{2}\right)^{2 s} \frac{\bar{D}_{4}^{2} R^{4}}{j^{4} \pi^{4}} t^{-2 \beta} \frac{j^{4}}{\underline{E}^{2}}\left(\int_{0}^{T}(T-s)^{\beta-1} E_{\beta, \beta}\left(-\left(\frac{j \pi}{R}\right)^{2}(T-s)^{\beta}\right)\left\langle G(\cdot, s), \psi_{j}\right\rangle d s\right)^{2} \\
& \quad \leq \xi_{1}^{2} \mathcal{F}_{1}^{2} \mathcal{F}_{2}^{2} t^{-2 \beta} \sum_{j=1}^{+\infty}\left(j^{2}\right)^{2 s-2 \gamma}\left(\int_{0}^{T}(T-s)^{\beta-1-\beta \gamma}\left\langle G(\cdot, s), \psi_{j}\right\rangle d s\right)^{2} .
\end{aligned}
$$

Using the Hölder inequality, we get the following estimate:

$$
\begin{aligned}
& \left(\int_{0}^{T}(T-s)^{\beta-1-\beta \gamma}\left\langle G(\cdot, s), \psi_{j}\right\rangle d s\right)^{2} \\
& \quad \leq\left(\int_{0}^{T}(T-s)^{\beta-1-\beta \gamma} d s\right)\left(\int_{0}^{T}(T-s)^{\beta-1-\beta \gamma}\left|\left\langle G(\cdot, s), \psi_{j}\right\rangle\right|^{2} d s\right) \\
& \quad \leq\left(\frac{T^{\beta-\beta \gamma}}{\beta-\beta \gamma}\right)\left(\int_{0}^{T}(T-s)^{\beta-1-\beta \gamma}\left|\left\langle G(\cdot, s), \psi_{j}\right\rangle\right|^{2} d s\right) .
\end{aligned}
$$

From (3.20) and (3.21), one has the following bound for the second term $\mathcal{A}_{2}(\cdot, t)$ :

$$
\begin{aligned}
& \left\|\mathcal{A}_{2}(\cdot, t)\right\|_{\mathcal{H}^{s}\left([0, R] ; r^{2}\right)}^{2} \\
& \quad \leq \xi_{1}^{2} \mathcal{F}_{1}^{2} \mathcal{F}_{2}^{2}\left(\frac{T^{\beta-\beta \gamma}}{\beta-\beta \gamma}\right)^{2} t^{-2 \beta}\left(\int_{0}^{T}(T-s)^{\beta-1-\beta \gamma} \sum_{j=1}^{+\infty}\left(j^{2}\right)^{2 s-2 \gamma}\left|\left\langle G(\cdot, s), \psi_{j}\right\rangle\right|^{2} d s\right) \\
& \quad \leq \xi_{1}^{2} \mathcal{F}_{1}^{2} \mathcal{F}_{2}^{2}\left(\frac{T^{\beta-\beta \gamma}}{\beta-\beta \gamma}\right)^{2} t^{-2 \beta}\|G\|_{L^{\infty}\left(0, T ; \mathcal{H}^{s-\gamma}\right)\left([0, R] ; r^{2}\right)}^{2} .
\end{aligned}
$$

From (3.22), we conclude that

$$
\left\|\mathcal{A}_{2}(\cdot, t)\right\|_{\mathcal{H}^{s}\left([0, R] ; r^{2}\right)} \leq \xi_{1} \mathcal{F}_{1} \mathcal{F}_{2}\left(\frac{T^{\beta-\beta \gamma}}{\beta-\beta \gamma}\right) t^{-\beta}\|G\|_{L^{\infty}\left(0, T ; \mathcal{H}^{s-\gamma}\left([0, R] ; r^{2}\right)\right)} .
$$


Step 3: Estimate for $\left\|\mathcal{A}_{3}(\cdot, t)\right\|_{\mathcal{H}^{s}\left([0, R] ; r^{2}\right)}$, by using (3.19), we receive

$$
\begin{aligned}
& \left\|\mathcal{A}_{3}(\cdot, t)\right\|_{\mathcal{H}^{s}\left([0, R] ; r^{2}\right)}^{2} \\
& \quad \leq \xi_{2}^{2} \mathcal{F}_{1}^{2} \sum_{j=1}^{+\infty}\left(j^{2}\right)^{2 s-2} t^{-2 \beta}\left(\int_{0}^{T}\left(\int_{0}^{t}(t-s)^{\beta-1} E_{\beta, \beta}\left(-\left(\frac{j \pi}{R}\right)^{2}(t-s)^{\beta}\right)\left\langle G(\cdot, s), \psi_{j}\right\rangle\right) d t\right)^{2} .
\end{aligned}
$$

By applying the Hölder inequality and denoting $0<\gamma<\min \left\{1, \frac{1}{2 \beta}\right\}$, we find the following bound:

$$
\begin{aligned}
& \left|\int_{0}^{T}\left(\int_{0}^{t}(t-s)^{\beta-1} E_{\beta, \beta}\left(-\left(\frac{j \pi}{R}\right)^{2}(t-s)^{\beta}\right)\left\langle G(\cdot, s), \psi_{j}\right\rangle\right) d t\right| \\
& \quad \leq \bar{D}_{6}\left(j^{2}\right)^{-\gamma} \int_{0}^{T}\left(\int_{0}^{t}(t-s)^{-2 \gamma \beta} d s\right)^{1 / 2}\left(\int_{0}^{t}\left|\left\langle G(\cdot, s), \psi_{j}\right\rangle\right|^{2} d s\right)^{1 / 2} d t \\
& \quad \leq T \bar{D}_{6}\left(j^{2}\right)^{-\gamma}\left(\frac{T^{1-2 \gamma \beta}}{1-2 \gamma \beta}\right)^{\frac{1}{2}} \int_{0}^{T}\left(\int_{0}^{t}\left|\left\langle G(\cdot, s), \psi_{j}\right\rangle\right|^{2} d s\right)^{1 / 2} d t \\
& \quad \leq T \bar{D}_{6}\left(\frac{T^{1-2 \gamma \beta}}{1-2 \gamma \beta}\right)^{\frac{1}{2}}\left(\int_{0}^{T}\left(j^{2}\right)^{-\gamma}\left|\left\langle G(\cdot, s), \psi_{j}\right\rangle\right|^{2} d s\right)^{1 / 2} .
\end{aligned}
$$

From (3.24), with $\mathcal{F}_{3}^{2}=\xi_{2}^{2} \mathcal{F}_{1}^{2} T \bar{D}_{6}^{2}\left(\frac{T^{1-2 \gamma \beta}}{1-2 \gamma \beta}\right)$, by two above observations, we deduce that

$$
\begin{aligned}
\left\|\mathcal{A}_{3}(\cdot, t)\right\|_{\mathcal{H}^{s}\left([0, R] ; r^{2}\right)}^{2} & \leq \mathcal{F}_{3}^{2} t^{-2 \beta} \sum_{j=1}^{+\infty}\left(\int_{0}^{T}\left(j^{2}\right)^{2 s-2-2 \gamma}\left|\left\langle G(\cdot, s), \psi_{j}\right)\right|^{2} d s\right) \\
& \leq \mathcal{F}_{3}^{2} t^{-2 \beta}\|G\|_{L^{2}\left(0, T ; \mathcal{H}^{s-1-\gamma}\left([0, R] ; r^{2}\right)\right)}^{2} .
\end{aligned}
$$

Step 4: Estimate for $\left\|\mathcal{A}_{4}(\cdot, t)\right\|_{\mathcal{H}^{s}\left([0, R] ; r^{2}\right)}$, using (3.19) and by applying the Hölder inequality, $\left\|\mathcal{A}_{4}(\cdot, t)\right\|_{\mathcal{H}^{s}\left([0, R] ; r^{2}\right)}$ can be bounded as follows:

$$
\begin{aligned}
& \left\|\mathcal{A}_{4}(\cdot, t)\right\|_{\mathcal{H}^{s}\left([0, R] ; r^{2}\right)}^{2} \\
& \quad \leq \sum_{j=1}^{+\infty}\left(\int_{0}^{t}(t-s)^{\beta-1} E_{\beta, \beta}\left(-\left(\frac{j \pi}{R}\right)^{2}(t-s)^{\beta}\right)\left\langle G(\cdot, s), \psi_{j}\right\rangle d s\right)^{2} \\
& \quad \leq \mathcal{F}_{2}^{2} \sum_{j=1}^{+\infty}\left(j^{2}\right)^{2 s-2 \gamma}\left(\int_{0}^{t}(t-s)^{\beta-1-\beta \gamma}\left\langle G(\cdot, s), \psi_{j}\right\rangle d s\right)^{2} \\
& \quad \leq \mathcal{F}_{2}^{2} \sum_{j=1}^{+\infty}\left(j^{2}\right)^{2 s-2 \gamma}\left(\int_{0}^{t}(t-s)^{\beta-1-\beta \gamma} d s\right) \times\left(\int_{0}^{t}(t-s)^{\beta-1-\beta \gamma}\left|\left\langle G(\cdot, s), \psi_{j}\right\rangle\right|^{2} d s\right) \\
& \quad \leq \mathcal{F}_{2}^{2}\left(\frac{T^{\beta-\beta \gamma}}{\beta-\beta \gamma}\right)\left(\int_{0}^{t}(t-s)^{\beta-1-\beta \gamma} \sum_{j=1}^{+\infty}\left(j^{2}\right)^{2 s-2 \gamma}\left|\left\langle G(\cdot, s), \psi_{j}\right\rangle\right|^{2} d s\right) \\
& \quad \leq \mathcal{F}_{2}^{2}\left(\frac{T^{\beta-\beta \gamma}}{\beta-\beta \gamma}\right)^{2}\|G\|_{L^{\infty}\left(0, T ; \mathcal{H}^{s-\gamma}\left([0, R] ; r^{2}\right)\right)}^{2} .
\end{aligned}
$$


Combining four steps as above, we conclude that

$$
\begin{aligned}
& \|u(\cdot, t)\|_{\mathcal{H}^{s}\left([0, R] ; r^{2}\right)} \\
& \quad \leq \mathcal{F}_{1} t^{-\beta}\|f\|_{\mathcal{H}^{s}\left([0, R] ; r^{2}\right)}+\xi_{1} \mathcal{F}_{1} \mathcal{F}_{2} \frac{T^{\beta-\beta \gamma}}{\beta-\beta \gamma} t^{-\beta}\|G\|_{L^{\infty}\left(0, T ; \mathcal{H}^{s-\gamma}\left([0, R] ; r^{2}\right)\right)} \\
& \quad+\mathcal{F}_{3} t^{-\beta}\|G\|_{L^{2}\left(0, T ; \mathcal{H}^{s-1-\gamma}\left([0, R] ; r^{2}\right)\right)}+\mathcal{F}_{2}\left(\frac{T^{\beta-\beta \gamma}}{\beta-\beta \gamma}\right)\|G\|_{L^{\infty}\left(0, T ; \mathcal{H}^{s-\gamma}\left([0, R] ; r^{2}\right)\right)} .
\end{aligned}
$$

From (3.28), by choosing $1<p<\frac{1}{\beta}$, then the integral $\int_{0}^{T} t^{-\beta p} d t$ is convergent, we have a comment as follows:

$$
\begin{aligned}
\|u\|_{L^{p}\left(0, T ; \mathcal{H}^{s}\left([0, R] ; r^{2}\right)\right)}= & \left(\int_{0}^{T}\|u(\cdot, t)\|_{\mathcal{H}^{s}\left([0, R] ; r^{2}\right)}^{p} d t\right)^{\frac{1}{p}} \\
\leq & \left(\int_{0}^{T} t^{-\beta p} d t\right)^{\frac{1}{p}} \mathcal{F}_{1}\|f\|_{\mathcal{H}^{s}\left([0, R] ; r^{2}\right)} \\
& +\left(\int_{0}^{T} t^{-\beta p} d t\right)^{\frac{1}{p}} \xi_{1} \mathcal{F}_{1} \mathcal{F}_{2} \frac{T^{\beta-\beta \gamma}}{\beta-\beta \gamma}\|G\|_{L^{\infty}\left(0, T ; \mathcal{H}^{s-\gamma}\left([0, R] ; r^{2}\right)\right)} \\
& +\left(\int_{0}^{T} t^{-\beta p} d t\right)^{\frac{1}{p}} \mathcal{F}_{3}\|G\|_{L^{2}\left(0, T ; \mathcal{H}^{s-1-\gamma}\left([0, R] ; r^{2}\right)\right)} \\
& +\left(\int_{0}^{T} d t\right)^{\frac{1}{p}} \mathcal{F}_{2}\left(\frac{T^{\beta-\beta \gamma}}{\beta-\beta \gamma}\right)\|G\|_{L^{\infty}\left(0, T ; \mathcal{H}^{s-\gamma}\left([0, R] ; r^{2}\right)\right)},
\end{aligned}
$$

this implies that

$$
\begin{aligned}
\|u\|_{L^{p}\left(0, T ; \mathcal{H}^{s}\left([0, R] ; r^{2}\right)\right)} \lesssim & \|f\|_{\mathcal{H}^{s}\left([0, R] ; r^{2}\right)}+\|G\|_{L^{\infty}\left(0, T ; \mathcal{H}^{s-\gamma}\left([0, R] ; r^{2}\right)\right)} \\
& +\|G\|_{L^{2}\left(0, T ; \mathcal{H}^{s-1-\gamma}\left([0, R] ; r^{2}\right)\right)}
\end{aligned}
$$

\section{III-posedness and conditional stability of problem (1.1)}

\subsection{The ill-posedness of problem (1.1)}

Theorem 4.1 The inverse problem (1.1) is ill-posed in the case $t=0$.

Proof A linear operator $\mathcal{K}: L^{2}\left([0, R] ; r^{2}\right) \rightarrow L^{2}\left([0, R] ; r^{2}\right)$ is as follows:

$$
\begin{aligned}
\mathcal{K} \ell(r) & =\sum_{j=1}^{+\infty}\left[\xi_{1} E_{\beta, 1}\left(-\left(\frac{j \pi}{R}\right)^{2} T^{\beta}\right)+\xi_{2} \int_{0}^{T} E_{\beta, 1}\left(-\left(\frac{j \pi}{R}\right)^{2} t^{\beta}\right) d t\right]\left\langle\ell, \psi_{j}\right\rangle \psi_{j}(r) \\
& =\int_{\Omega} p(r, v) \ell(v) d v,
\end{aligned}
$$

where

$$
p(r, v)=\sum_{j=1}^{+\infty}\left[\xi_{1} E_{\beta, 1}\left(-\left(\frac{j \pi}{R}\right)^{2} T^{\beta}\right)+\xi_{2} \int_{0}^{T} E_{\beta, 1}\left(-\left(\frac{j \pi}{R}\right)^{2} t^{\beta}\right) d t\right] \psi_{k}(r) \psi_{k}(v) .
$$


It is obvious that $\ell(r, z)=\ell(z, r)$, we know $\mathcal{K}$ is a self-adjoint operator. Next, we are going to prove its compactness and consider the finite rank operators as follows:

$$
\mathcal{K}_{N} \ell(r)=\sum_{j=1}^{N}\left[\xi_{1} E_{\beta, 1}\left(-\left(\frac{j \pi}{R}\right)^{2} T^{\beta}\right)+\xi_{2} \int_{0}^{T} E_{\beta, 1}\left(-\left(\frac{j \pi}{R}\right)^{2} t^{\beta}\right) d t\right]\left\langle\ell, \psi_{j}\right\rangle \psi_{j}(r) .
$$

From (4.2), using the inequality $(a+b)^{2} \leq 2\left(a^{2}+b^{2}\right), a, b \geq 0$, we have

$$
\left\|\mathcal{K}_{N} \ell-\mathcal{K} \ell\right\|_{L^{2}\left([0, R] ; r^{2}\right)}^{2} \leq \sum_{j=N+1}^{+\infty} \frac{\bar{E}^{2}}{j^{4}}\left|\left\langle\ell, \psi_{j}\right\rangle\right|^{2} \leq \frac{\bar{E}^{2}}{N^{4}}\|\ell\|_{L^{2}\left([0, R] ; r^{2}\right)}^{2}
$$

Therefore, $\left\|\mathcal{K}_{N} \ell-\mathcal{K} \ell\right\|_{L^{2}\left([0, R] ; r^{2}\right)}$ in the sense of operator norm in $L\left(L^{2}\left([0, R] ; r^{2}\right) ; L^{2}([0, R]\right.$; $\left.\left.r^{2}\right)\right)$ as $N \rightarrow \infty$. Also, $\mathcal{K}$ is a compact operator. Next, the SVDs for the linear self-adjoint compact operator $\mathcal{K}$ is $\mathcal{K} \ell=f$, and by Kirsch [34], we conclude that the problem is illposed. Next, we continue to give an example for ill-posedness. In here, we assume $f \in$ $L^{2}\left([0, R] ; r^{2}\right)$ and $G \in L^{\infty}\left(0, T ; L^{2}\left([0, R] ; r^{2}\right)\right)$ and $f=G=0$. In here, we choose

$$
f_{k}(r)=\frac{\psi_{k}(r)}{k}, \quad G_{k}(r, s)=(T-s)^{2} E_{\beta, 1}\left(-\left(\frac{k \pi}{R}\right)^{2}(T-s)^{\beta}\right) \psi_{k}(r), \quad 0<s<T .
$$

Let us choose input final data $f=0$, we know that an error in the $L^{2}\left([0, R] ; r^{2}\right)$ norm between two input final data is as follows:

$$
\begin{aligned}
& \left\|f_{k}-f\right\|_{L^{2}\left([0, R] ; r^{2}\right)}=\left\|\frac{\psi_{k}(\cdot)}{k}\right\|_{L^{2}\left([0, R] ; r^{2}\right)}=\frac{1}{k}, \\
& \text { this yields } \lim _{k \rightarrow \infty}\left\|f_{k}-f\right\|_{L^{2}\left([0, R] ; r^{2}\right)}=\lim _{k \rightarrow \infty} \frac{1}{k}=0 .
\end{aligned}
$$

On the other hand, because of $\beta \in(0,1)$, one has

$$
\begin{aligned}
\left\|G_{k}-G\right\|_{L^{\infty}\left(0, T ; L^{2}\left([0, R] ; r^{2}\right)\right.} & \leq\left\|\left(\frac{T^{2} \bar{D}_{4}}{1+\left(\frac{k \pi}{R}\right)^{2} T^{\beta}}\right)\right\|_{L^{\infty}\left(0, T ; L^{2}\left([0, R] ; r^{2}\right)\right.} \\
& \leq\left(\frac{R^{2}}{\pi^{2}}\right)\left(T^{2} \bar{D}_{4}\right) \frac{1}{k^{2}} \rightarrow 0 \quad \text { as } k \rightarrow \infty .
\end{aligned}
$$

Combining (4.5) and (4.6) yields that

$$
\left\|f_{k}-f\right\|_{L^{2}\left([0, R] ; r^{2}\right)}+\left\|G_{k}-G\right\|_{L^{\infty}\left(0, T ; L^{2}[0, R] ; r^{2}\right)} \rightarrow 0, \quad \text { if } k \rightarrow \infty .
$$

Setting $u_{k}(r, 0)$ is the solution of $(1.1)$, we obtain

$$
u_{k}(r, 0)=\frac{f_{k}(r)-\int_{0}^{T}(T-s)^{\beta-1} E_{\beta, \beta}\left(-\left(\frac{k \pi}{R}\right)^{2}(T-s)^{\beta}\right) G_{k}(r, s) d s}{\xi_{1} E_{\beta, 1}\left(-\left(\frac{k \pi}{R}\right)^{2} T^{\beta}\right)+\xi_{2} \int_{0}^{T} E_{\beta, 1}\left(-\left(\frac{k \pi}{R}\right)^{2} t^{\beta}\right) d t} .
$$


Using the inequality $|a-b|>|a|-|b|$, we know that

$$
\begin{aligned}
\left|u_{k}(\cdot, 0)\right| \geq & \left|\frac{f_{k}}{\xi_{1} E_{\beta, 1}\left(-\left(\frac{k \pi}{R}\right)^{2} T^{\beta}\right)+\xi_{2} \int_{0}^{T} E_{\beta, 1}\left(-\left(\frac{k \pi}{R}\right)^{2} t^{\beta}\right) d t}\right| \\
& -\left|\frac{\int_{0}^{T}(T-s)^{\beta-1} E_{\beta, \beta}\left(-\left(\frac{k \pi}{R}\right)^{2}(T-s)^{\beta}\right) G_{k}(\cdot, s) d s}{\xi_{1} E_{\beta, 1}\left(-\left(\frac{k \pi}{R}\right)^{2} T^{\beta}\right)+\xi_{2} \int_{0}^{T} E_{\beta, 1}\left(-\left(\frac{k \pi}{R}\right)^{2} t^{\beta}\right) d t}\right| .
\end{aligned}
$$

From (4.9), we get

$$
\begin{gathered}
\left|u_{k}(\cdot, 0)\right|+\left|\frac{\int_{0}^{T}(T-s)^{\beta-1} E_{\beta, \beta}\left(-\left(\frac{k \pi}{R}\right)^{2}(T-s)^{\beta}\right) G_{k}(\cdot, s) d s}{\xi_{1} E_{\beta, 1}\left(-\left(\frac{k \pi}{R}\right)^{2} T^{\beta}\right)+\xi_{2} \int_{0}^{T} E_{\beta, 1}\left(-\left(\frac{k \pi}{R}\right)^{2} t^{\beta}\right) d t}\right| \\
\geq\left|\frac{f_{k}}{\xi_{1} E_{\beta, 1}\left(-\left(\frac{k \pi}{R}\right)^{2} T^{\beta}\right)+\xi_{2} \int_{0}^{T} E_{\beta, 1}\left(-\left(\frac{k \pi}{R}\right)^{2} t^{\beta}\right) d t}\right| .
\end{gathered}
$$

Applying the inequality $(a+b)^{2} \leq 2\left(a^{2}+b^{2}\right), \forall a, b \geq 0$ and Hölder's inequality, we get

$$
\begin{aligned}
& \left|\frac{f_{k}}{\xi_{1} E_{\beta, 1}\left(-\left(\frac{j \pi}{R}\right)^{2} T^{\beta}\right)+\xi_{2} \int_{0}^{T} E_{\beta, 1}\left(-\left(\frac{j \pi}{R}\right)^{2} t^{\beta}\right) d t}\right|^{2} \\
& \quad \leq 2\left|u_{k}(\cdot, 0)\right|^{2}+2 T \int_{0}^{T}\left|\frac{(T-s)^{\beta-1} E_{\beta, \beta}\left(-\left(\frac{k \pi}{R}\right)^{2}(T-s)^{\beta}\right) G_{k}(\cdot, s)}{\xi_{1} E_{\beta, 1}\left(-\left(\frac{k \pi}{R}\right)^{2} T^{\beta}\right)+\xi_{2} \int_{0}^{T} E_{\beta, 1}\left(-\left(\frac{k \pi}{R}\right)^{2} t^{\beta}\right) d t}\right|^{2} d s .
\end{aligned}
$$

Combining (4.4), (4.5), and (4.6), we obtain

$$
\begin{aligned}
\frac{k^{2}}{\bar{E}^{2}} \leq & 2\left\|u_{k}(\cdot, 0)\right\|_{L^{2}\left([0, R] ; r^{2}\right)}^{2} \\
& +\frac{2 T k^{4}}{\underline{E}^{2}} \int_{0}^{T}\left|(T-s)^{\beta+1} E_{\beta, \beta}\left(-\left(\frac{k \pi}{R}\right)^{2}(T-s)^{\beta}\right) E_{\beta, 1}\left(-\left(\frac{k \pi}{R}\right)^{2}(T-s)^{\beta}\right)\right|^{2} d s .
\end{aligned}
$$

From the aforementioned inequality, we get the following estimate:

$$
\frac{k^{2}}{\bar{E}^{2}} \leq 2\left\|u_{k}(\cdot, 0)\right\|_{L^{2}\left([0, R] ; r^{2}\right)}^{2}+\frac{2 T R^{8} \bar{D}_{4}^{4}}{\pi^{8} \underline{E}^{2}} \frac{1}{k^{4}} \int_{0}^{T}\left|(T-s)^{1-\beta}\right|^{2} d s .
$$

Hence, we come up with the estimate

$$
\frac{k^{2}}{\bar{E}^{2}} \leq 2\left\|u_{k}(\cdot, 0)\right\|_{L^{2}\left([0, R] ; r^{2}\right)}^{2}+\frac{1}{k^{4}} \frac{2 T R^{8} \bar{D}_{4}^{4}}{\pi^{8} \underline{E}^{2}} \int_{0}^{T}(T-s)^{2} d s,
$$

which allows us to give that

$$
\frac{k^{2}}{\bar{E}^{2}} \leq 2\left\|u_{k}(\cdot, 0)\right\|_{L^{2}\left([0, R] ; r^{2}\right)}^{2}+\frac{1}{k^{4}} \frac{2 T^{4} R^{8} \bar{D}_{4}^{4}}{\pi^{8} \underline{E}^{2}}
$$

Using the inequality $\sqrt{a^{2}+b^{2}} \leq a+b, \forall a, b \geq 0$, one has

$$
\frac{k}{\bar{E}} \leq \sqrt{2}\left\|u_{k}(\cdot, 0)\right\|_{L^{2}\left([0, R] ; r^{2}\right)}^{2}+\frac{1}{k^{2}} \sqrt{\frac{2 T^{4} R^{8} \bar{D}_{4}^{4}}{\pi^{8} \underline{E}^{2}}}
$$


Therefore, we have that

$$
\left\|u_{k}(\cdot, 0)\right\|_{L^{2}\left([0, R] ; r^{2}\right)} \geq \frac{k}{\sqrt{2 \bar{E}}}-\frac{1}{k^{2}} \sqrt{\frac{2 T^{4} R^{8} \bar{D}_{4}^{4}}{\pi^{8} \underline{E}^{2}}} \rightarrow \infty, \quad \text { if } k \rightarrow \infty
$$

Thus, problem (4.8) is, in general, ill-posed in the Hadamard sense in the $L^{2}\left([0, R] ; r^{2}\right)$ norm.

\subsection{The conditional stability of the solution for problem (1.1)}

Theorem 4.2 Let us assume that $\mathcal{C}$ is a positive constant such that $u(\cdot, 0) \in \mathcal{H}^{s}\left([0, R] ; r^{2}\right)$ for some $s>0$ satisfying the following a priori bound condition:

$$
\|u(\cdot, 0)\|_{\mathcal{H}^{s}\left([0, R] ; r^{2}\right)} \leq \mathcal{C}
$$

Then we have

$$
\|u(\cdot, 0)\|_{L^{2}\left([0, R] ; r^{2}\right)} \leq \mathcal{S}(s, f, G) \mathcal{C}^{\frac{1}{s+1}}
$$

where

$$
\overline{\mathcal{S}}(s, f, G)=\left(2\|f\|_{L^{2}\left([0, R] ; r^{2}\right)}^{2}+2 \bar{D}_{5}^{2}\|G\|_{L^{\infty}\left(0, T ; L^{2}\left([0, T] ; r^{2}\right)\right)}^{2}\right)^{\frac{s}{2(s+1)}} .
$$

Proof By using Parseval's equality, we obtain that

$$
\begin{aligned}
\|u(\cdot, 0)\|_{L^{2}\left([0, R] ; r^{2}\right)}^{2} & \\
= & \sum_{j=1}^{+\infty} \frac{\left|\left\langle f, \psi_{j}\right\rangle-\xi_{1} \int_{0}^{T}(T-s)^{\beta-1} E_{\beta, \beta}\left(-\left(\frac{j \pi}{R}\right)^{2}(T-s)^{\beta}\right)\left\langle G(\cdot, s), \psi_{j}\right\rangle d s\right|^{2}}{\left|\xi_{1} E_{\beta, 1}\left(-\left(\frac{j \pi}{R}\right)^{2} T^{\beta}\right)+\xi_{2} \int_{0}^{T} E_{\beta, 1}\left(-\left(\frac{j \pi}{R}\right)^{2} t^{\beta}\right) d t\right|^{2}} \\
\leq & \sum_{j=1}^{\infty}\left(\frac{\left|\left\langle f, \psi_{j}\right\rangle-\xi_{1} \int_{0}^{T}(T-s)^{\beta-1} E_{\beta, \beta}\left(-\left(\frac{j \pi}{R}\right)^{2}(T-s)^{\beta}\right)\left\langle G(\cdot, s), \psi_{j}\right\rangle d s\right|^{2}}{\left|\xi_{1} E_{\beta, 1}\left(-\left(\frac{j \pi}{R}\right)^{2} T^{\beta}\right)+\xi_{2} \int_{0}^{T} E_{\beta, 1}\left(-\left(\frac{j \pi}{R}\right)^{2} t^{\beta}\right) d t\right|^{2 s+2}}\right)^{\frac{1}{s+1}} \\
& \times \sum_{j=1}^{\infty}\left(2\left|\left\langle f, \psi_{j}\right\rangle\right|^{2}+2 \xi_{1}^{2}\left|\int_{0}^{T}(T-s)^{\beta-1} E_{\beta, \beta}\left(-\left(\frac{j \pi}{R}\right)^{2}(T-s)^{\beta}\right)\left\langle G(\cdot, s), \psi_{j}\right\rangle d s\right|^{2}\right)^{\frac{s}{s+1}} \\
\leq & \mathcal{Q}_{1}^{\frac{1}{s+1}} \times \mathcal{Q}_{2}^{\frac{s}{s+1}} .
\end{aligned}
$$

The term $Q_{1}$ can be estimated by using Lemma 2.4 as follows:

$$
\begin{aligned}
\mathcal{Q}_{1} & =\sum_{j=1}^{+\infty}\left(\frac{\left|\left\langle f, \psi_{j}\right\rangle-\xi_{1} \int_{0}^{T}(T-s)^{\beta-1} E_{\beta, \beta}\left(-\left(\frac{j \pi}{R}\right)^{2}(T-s)^{\beta}\right)\left\langle G(\cdot, s), \psi_{j}\right\rangle d s\right|^{2}}{\left|\xi_{1} E_{\beta, 1}\left(-\left(\frac{j \pi}{R}\right)^{2} T^{\beta}\right)+\xi_{2} \int_{0}^{T} E_{\beta, 1}\left(-\left(\frac{j \pi}{R}\right)^{2} t^{\beta}\right) d t\right|^{2 s+2}}\right) \\
& \leq \sum_{j=1}^{+\infty} \frac{\left(j^{2}\right)^{2 s}}{\underline{E}^{2 s}}\left|u_{j}(\cdot, 0)\right|^{2}=\frac{1}{\underline{E}^{2 s}}\|u(\cdot, 0)\|_{\mathcal{H}^{s}\left([0, R] ; r^{2}\right)^{.}}^{2}
\end{aligned}
$$


The term $\mathcal{Q}_{2}$ can be bounded as follows:

$$
\begin{aligned}
\mathcal{Q}_{2}^{2} & \leq 2 \sum_{j=1}^{+\infty}\left|\left\langle f, \psi_{j}\right\rangle\right|^{2}+2 \xi_{1}^{2} \sum_{j=1}^{+\infty}\left|\int_{0}^{T}(T-s)^{\beta-1} E_{\beta, \beta}\left(-\left(\frac{j \pi}{R}\right)^{2}(T-s)^{\beta}\right)\left\langle G(\cdot, s), \psi_{j}\right\rangle d s\right|^{2} \\
& \leq 2\|f\|_{L^{2}\left([0, R] ; r^{2}\right)}^{2}+2 \bar{D}_{5}^{2}\|G\|_{L^{\infty}\left(0, T ; L^{2}\left([0, T] ; r^{2}\right)\right)^{2}}^{2}
\end{aligned}
$$

Combining (4.21)-(4.23), we complete the proof.

\section{The fractional Tikhonov method and convergence rate}

\subsection{The fractional Tikhonov method}

In this section, we apply the fractional Tikhonov method given by Morigi [35]. From now on, we denote

$$
\Theta_{\beta, j}^{\xi_{1}, \xi_{2}}(\pi, R, T)=\xi_{1} E_{\beta, 1}\left(-\left(\frac{j \pi}{R}\right)^{2} T^{\beta}\right)+\xi_{2} \int_{0}^{T} E_{\beta, 1}\left(-\left(\frac{j \pi}{R}\right)^{2} t^{\beta}\right) d t
$$

we propose the following regularized solution with exact data $(f, G)$ :

$$
\mathcal{P}_{\alpha, \delta}(r)=\sum_{j=1}^{+\infty}\left|\Theta_{\beta, j}^{\xi_{1}, \xi_{2}}(\pi, R, T)\right|^{-1}\left[\frac{\left|\Theta_{\beta, j}^{\xi_{1}, \xi_{2}}(\pi, R, T)\right|^{2}}{\left|\Theta_{\beta, j}^{\xi_{1}, \xi_{2}}(\pi, R, T)\right|^{2}+\alpha}\right]^{\delta}\left\langle\Xi, \psi_{j}\right\rangle \psi_{j}(r)
$$

in which

$$
\begin{aligned}
\Xi(r)= & \sum_{j=1}^{+\infty}\left\langle f, \psi_{j}\right\rangle \psi_{j}(r) \\
& -\xi_{1} \sum_{j=1}^{+\infty} \int_{0}^{T}(T-s)^{\beta-1} E_{\beta, \beta}\left(-\left(\frac{j \pi}{R}\right)^{2}(T-s)^{\beta}\right)\left\langle G(\cdot, s), \psi_{j}\right\rangle d s \psi_{j}(r) .
\end{aligned}
$$

However, if the measured data $\{f, G\}$ are noised by $\left\{f_{\epsilon}, G_{\epsilon}\right\}$, then we get

$$
\mathcal{P}_{\alpha, \delta}^{\epsilon}(r)=\sum_{j=1}^{+\infty}\left|\Theta_{\beta, j}^{\xi_{1}, \xi_{2}}(\pi, R, T)\right|^{-1}\left[\frac{\left|\Theta_{\beta, j}^{\xi_{1}, \xi_{2}}(\pi, R, T)\right|^{2}}{\left|\Theta_{\beta, j}^{\xi_{1}, \xi_{2}}(\pi, R, T)\right|^{2}+\alpha}\right]^{\delta}\left\langle\Xi^{\epsilon}, \psi_{j}\right\rangle \psi_{j}(r),
$$

in which

$$
\begin{aligned}
\Xi^{\epsilon}(r)= & \sum_{j=1}^{+\infty}\left\langle f^{\epsilon}, \psi_{j}\right\rangle \psi_{j}(r) \\
& -\xi_{1} \int_{0}^{T}(T-s)^{\beta-1} E_{\beta, \beta}\left(-\left(\frac{j \pi}{R}\right)^{2}(T-s)^{\beta}\right)\left\langle G^{\epsilon}(\cdot, s), \psi_{j}\right\rangle d s \psi_{j}(r),
\end{aligned}
$$

where $\delta \in\left(\frac{1}{2} ; 1\right]$ and $\alpha>0$. Noting that when $\delta=1$, the fractional Tikhonov method becomes a standard Tikhonov regularization. 
Lemma 5.1 Let $\delta \in\left(\frac{1}{2} ; 1\right]$, we have

$$
\sup _{j>0}\left|\Theta_{\beta, j}^{\xi_{1}, \xi_{2}}(\pi, R, T)\right|^{-1}\left[\frac{\left|\Theta_{\beta, j}^{\xi_{1}, \xi_{2}}(\pi, R, T)\right|^{2}}{\left|\Theta_{\beta, j}^{\xi_{1}, \xi_{2}}(\pi, R, T)\right|^{2}+\alpha}\right]^{\delta} \leq \mathcal{Z}_{\delta}^{\frac{1}{2}} \alpha^{-\frac{1}{2}},
$$

with $\mathcal{Z}$ depending on $\delta$.

Proof The proof of lemma can be found in [36].

\subsection{An a priori parameter choice rule}

Let us consider the operator

$$
\mathcal{Q}_{\beta}(\pi, R, T)(t) v:=\sum_{j=1}^{+\infty}\left|\Theta_{\beta, j}^{\xi_{1}, \xi_{2}}(\pi, R, T)\right|^{-1}\left[\frac{\left|\Theta_{\beta, j}^{\xi_{1}, \xi_{2}}(\pi, R, T)\right|^{2}}{\left|\Theta_{\beta, j}^{\xi_{1}, \xi_{2}}(\pi, R, T)\right|^{2}+\alpha}\right]^{\delta}\left\langle\nu, \psi_{j}\right\rangle \psi_{j}(r),
$$

for $v \in L^{2}\left([0, R] ; r^{2}\right)$ and $0 \leq t \leq T$.

By applying the fractional Tikhonov method, we can see that

$$
\begin{aligned}
& u_{\alpha, \delta}(r, 0) \\
& =\sum_{j=1}^{+\infty}\left|\Theta_{\beta, j}^{\xi_{1}, \xi_{2}}(\pi, R, T)\right|^{-1}\left[\frac{\left|\Theta_{\beta, j}^{\xi_{1}, \xi_{2}}(\pi, R, T)\right|^{2}}{\left|\Theta_{\beta, j}^{\xi_{1}, \xi_{2}}(\pi, R, T)\right|^{2}+\alpha}\right]^{\delta} \\
& \quad \times\left[\left\langle f, \psi_{j}\right\rangle-\xi_{1} \int_{0}^{T}(T-s)^{\beta-1} E_{\beta, \beta}\left(-\left(\frac{j \pi}{R}\right)^{2}(T-s)^{\beta}\right)\left\langle G(\cdot, s), \psi_{j}\right\rangle d s\right] \psi_{j}(r) .
\end{aligned}
$$

By choosing the regularization parameter $\alpha$, the following theorem gives that the choice $\alpha$ is valid by using suitable assumptions. In order to give error estimate, let us assume that $\|\Xi\|_{\mathcal{H}^{s}\left([0, R] ; r^{2}\right)} \leq \mathcal{C}$ for any $s>0$, where $\mathcal{C}$ is a positive constant. Before going to the main theorem, we have auxiliary lemmas as follows.

Lemma 5.2 For some positive constant, we get

$$
\frac{\alpha \varsigma^{2-2 k}}{\mathcal{N}^{2}+\alpha \varsigma^{2}} \leq \begin{cases}\frac{k^{k}(1-k)^{1-k}}{\mathcal{N}^{2 k}} \alpha^{k}, & \text { if } 0<k<1 \\ \frac{1}{\mathcal{N}^{2} s^{2 k-2}} \alpha, & \text { if } k \geq 1\end{cases}
$$

Proof This lemma is proven similarly [36].

Theorem 5.3 Let $f \in L^{2}\left([0, R] ; r^{2}\right)$ and $G \in L^{\infty}\left([0, T] ; L^{2}\left([0, R] ; r^{2}\right)\right)$, inside $\Theta$ performed as in the digital formula (5.2), and if we choose the parameter regularization

$$
\alpha= \begin{cases}\left(\frac{\epsilon}{\mathcal{C}}\right)^{\frac{2}{s+1}}, & \text { if } 0<s<1 \\ \left(\frac{\epsilon}{\mathcal{C}}\right), & \text { if } s \geq 1\end{cases}
$$

then it gives:

- If $0<s<1$, then we have

$$
\left\|u_{\alpha, \delta}^{\epsilon}(\cdot, 0)-u(\cdot, 0)\right\|_{L^{2}\left([0, R] ; r^{2}\right)} \text { is of order } \epsilon^{\frac{s}{s+1}} \text {. }
$$


- If $s \geq 1$, then we have

$$
\left\|u_{\alpha, \delta}^{\epsilon}(\cdot, 0)-u(\cdot, 0)\right\|_{L^{2}\left([0, R] ; r^{2}\right)} \text { is of order } \epsilon^{\frac{1}{2}}
$$

Proof From the triangle inequality, we have

$$
\begin{aligned}
\left\|u_{\alpha, \delta}^{\epsilon}(\cdot, 0)-u(\cdot, 0)\right\|_{L^{2}\left([0, R] ; r^{2}\right)} \leq & \left\|u_{\alpha, \delta}^{\epsilon}(\cdot, 0)-u_{\alpha, \delta}(\cdot, 0)\right\|_{L^{2}\left([0, R] ; r^{2}\right)} \\
& +\left\|u_{\alpha, \delta}(\cdot, 0)-u(\cdot, 0)\right\|_{L^{2}\left([0, R] ; r^{2}\right)} .
\end{aligned}
$$

First of all, we have the estimate $\left\|u_{\alpha, \delta}^{\epsilon}(\cdot, 0)-u_{\alpha, \delta}(\cdot, 0)\right\|_{L^{2}\left([0, R] ; r^{2}\right)}$. Now, using the inequality $(a+b)^{2} \leq 2\left(a^{2}+b^{2}\right)$ gives

$$
\begin{aligned}
& \left\|u_{\alpha, \delta}^{\epsilon}(\cdot, 0)-u_{\alpha, \delta}(\cdot, 0)\right\|_{L^{2}\left([0, R] ; r^{2}\right)}^{2} \\
& \leq 2 \sum_{j=1}^{+\infty}\left|\Theta_{\beta, j}^{\xi_{1}, \xi_{2}}(\pi, R, T)\right|^{-2}\left[\frac{\left|\Theta_{\beta, j}^{\xi_{1}, \xi_{2}}(\pi, R, T)\right|^{2}}{\left|\Theta_{\beta, j}^{\xi_{1}, \xi_{2}}(\pi, R, T)\right|^{2}+\alpha}\right]^{2 \delta} \\
& \quad \times\left(\left|\left\langle f-f^{\epsilon}, \psi_{j}\right\rangle\right|^{2}\right. \\
& \left.\quad+\xi_{1}^{2}\left|\int_{0}^{T}(T-s)^{\beta-1} E_{\beta, \beta}\left(-\left(\frac{j \pi}{R}\right)^{2}(T-s)^{\beta}\right)\left\langle G(\cdot, s)-G^{\epsilon}(\cdot, s), \psi_{j}\right\rangle d s\right|^{2}\right) .
\end{aligned}
$$

From (5.12), applying Lemma 2.3 and the estimate of (1.3), it is easy to see that

$$
\begin{aligned}
\left\|u_{\alpha, \delta}^{\epsilon}(\cdot, 0)-u_{\alpha, \delta}(\cdot, 0)\right\|_{L^{2}\left([0, R] ; r^{2}\right)}^{2} \leq & 2 \mathcal{Z}_{\delta} \alpha^{-1}\left(\left\|f-f^{\epsilon}\right\|_{L^{2}\left([0, R] ; r^{2}\right)}^{2}\right. \\
& \left.+\xi_{1}^{2} \bar{D}_{5}^{2}\left\|G^{\epsilon}-G\right\|_{L^{\infty}\left(0, T ; L^{2}\left([0, R] ; r^{2}\right)\right)}^{2}\right) .
\end{aligned}
$$

Hence, we conclude that

$$
\left\|u_{\alpha, \delta}^{\epsilon}(\cdot, 0)-u_{\alpha, \delta}(\cdot, 0)\right\|_{L^{2}\left([0, R] ; r^{2}\right)} \leq 2 \mathcal{Z}_{\delta}^{\frac{1}{2}} \alpha^{-\frac{1}{2}} \epsilon\left[1+\xi_{1}^{2} \bar{D}_{5}^{2}\right]^{\frac{1}{2}} .
$$

Moreover, with $\left\langle\Xi, \psi_{j}\right\rangle$ defined in (5.2), we also get

$$
\begin{aligned}
& \left\|u_{\alpha, \delta}(\cdot, 0)-u(\cdot, 0)\right\|_{L^{2}\left([0, R] ; r^{2}\right)}^{2} \\
& \quad \leq\left.\sum_{j=1}^{+\infty}\right|^{2}\left|\Theta_{\beta, j}^{\xi_{1}, \xi_{2}}(\pi, R, T)\right|^{-2}\left[1-\frac{\left|\Theta_{\beta, j}^{\xi_{1}, \xi_{2}}(\pi, R, T)\right|^{2}}{\left|\Theta_{\beta, j}^{\xi_{1}, \xi_{2}}(\pi, R, T)\right|^{2}+\alpha}\right]^{2 \delta}\left|\left\langle\Xi, \psi_{j}\right\rangle\right|^{2} .
\end{aligned}
$$

From the definition of $\|\Xi(\cdot)\|_{\mathcal{H}^{s}\left([0, R] ; r^{2}\right)} \leq \mathcal{C}$, for any $s>0, \delta \in\left(\frac{1}{2}, 1\right]$, we obtain

$$
\begin{aligned}
\left\|u_{\alpha, \delta}(\cdot, 0)-u(\cdot, 0)\right\|_{L^{2}\left([0, R] ; r^{2}\right)}^{2} & \leq \sum_{j=1}^{+\infty}\left[\frac{\alpha\left(j^{2}\right)^{-2 s}}{\left|\Theta_{\beta, j}^{\xi_{1}, \xi_{2}}(\pi, R, T)\right|^{2}+\alpha}\right] \frac{\left(j^{2}\right)^{2 s}\left|\left\langle\Xi, \psi_{j}\right\rangle\right|^{2}}{\left|\Theta_{\beta, j}^{\xi_{1}, \xi_{2}}(\pi, R, T)\right|^{2}} \\
& \leq \sup _{j \geq 1}\left[\frac{\alpha\left(j^{2}\right)^{-2 s}}{\left|\Theta_{\beta, j}^{\xi_{1}, \xi_{2}}(\pi, R, T)\right|^{2}+\alpha}\right] \mathcal{C}^{2} \\
& \leq \sup _{j \geq 1}\left[\frac{\alpha\left(j^{2}\right)^{2-2 s}}{\underline{E}^{2}+\alpha j^{4}}\right] \mathcal{C}^{2} .
\end{aligned}
$$


Applying Lemma 5.2 and Lemma 2.4, where we used $\left|\Theta_{\beta, j}^{\xi_{1}, \xi_{2}}(\pi, R, T)\right|^{2} \geq \frac{E^{2}}{j^{4}}$, we get

$$
\begin{aligned}
\sup _{j \geq 1}\left[\frac{\alpha\left(j^{2}\right)^{-2 s}}{\left|\Theta_{\beta, j}^{\xi_{1}, \xi_{2}}(\pi, R, T)\right|^{2}+\alpha}\right] & \leq \sup _{j \geq 1}\left[\frac{\alpha\left(j^{2}\right)^{2-2 s}}{\underline{E}^{2}+\alpha j^{4}}\right] \\
& \leq \begin{cases}\frac{s^{s}(1-s)^{1-s}}{\underline{E}^{2 s}} \alpha^{s}, & \text { if } 0<s<1, \\
\frac{1}{\underline{E}^{2}} \alpha, & \text { if } s \geq 1 .\end{cases}
\end{aligned}
$$

Combining (5.15), (5.16), and (5.17), we obtain

$$
\left\|u_{\alpha, \delta}(\cdot, 0)-u(\cdot, 0)\right\|_{L^{2}\left([0, R] ; r^{2}\right)}^{2} \leq \begin{cases}\frac{s^{s}(1-s)^{1-s}}{\underline{E}^{2 s}} \mathcal{C}^{2} \alpha^{s}, & \text { if } 0<s<1 \\ \frac{1}{\underline{E}^{2}} \mathcal{C}^{2} \alpha, & \text { if } s \geq 1 .\end{cases}
$$

From (5.14) and (5.18), combining the inequality $\sqrt{a^{2}+b^{2}} \leq a+b, \forall a, b \geq 0$, we deduce that

$$
\begin{aligned}
\left\|u_{\alpha, \delta}^{\epsilon}(\cdot, 0)-u(\cdot, 0)\right\|_{L^{2}\left([0, R] ; r^{2}\right)} \leq & 2 \mathcal{Z}_{\delta}^{\frac{1}{2}} \alpha^{-\frac{1}{2}} \epsilon\left(1+\xi_{1}^{2} \bar{D}_{5}^{2}\right)^{\frac{1}{2}} \\
& + \begin{cases}\left(\frac{s^{s}(1-s)^{1-s}}{\underline{E}^{2 s}}\right)^{\frac{1}{2}} \mathcal{C} \alpha^{\frac{s}{2}}, & \text { if } 0<s<1, \\
\frac{1}{\underline{E}} \mathcal{C} \alpha^{\frac{1}{2}}, & \text { if } s \geq 1 .\end{cases}
\end{aligned}
$$

Choose the regularization parameter $\alpha$ as

$$
\alpha= \begin{cases}\left(\frac{\epsilon}{\mathcal{C}}\right)^{\frac{2}{s+1}}, & \text { if } 0<s<1, \\ \left(\frac{\epsilon}{\mathcal{C}}\right), & \text { if } s \geq 1,\end{cases}
$$

then we have:

- If $0<s<1$, then we have the following estimate:

$$
\left\|u_{\alpha, \delta}^{\epsilon}(\cdot, 0)-u(\cdot, 0)\right\|_{L^{2}\left([0, R] ; r^{2}\right)} \text { is of order } \epsilon^{\frac{s}{s+1}}
$$

- If $s \geq 1$, then we have the following estimate:

$$
\left\|u_{\alpha, \delta}^{\epsilon}(\cdot, 0)-u(\cdot, 0)\right\|_{L^{2}\left([0, R] ; r^{2}\right)} \text { is of order } \epsilon^{\frac{1}{2}} .
$$

The proof is completed.

\subsection{An a posteriori parameter choice rule}

In this subsection, considering the choice of the a posteriori regularization parameter in Morozov's discrepancy principle, [37] we choose the regularization parameter $\alpha$ such that

$$
\left\|\mathcal{K} \mathcal{P}_{\alpha, \delta}^{\epsilon}-\Xi^{\epsilon}\right\|_{L^{2}\left([0, R] ; r^{2}\right)}=\zeta \epsilon
$$

where $\left\|\Xi^{\epsilon}\right\|_{L^{2}\left([0, R] ; r^{2}\right)} \geq \zeta \epsilon, \zeta>1$ depends on $\epsilon$. 
Lemma 5.4 For some positive constants $k, \alpha, \mathcal{N}, \varsigma$, we get

$$
\frac{\alpha \varsigma^{-k+1}}{\mathcal{N}^{2}+\alpha \varsigma^{2}} \leq \begin{cases}\frac{2^{-1}(k+1)^{\frac{k+1}{2}}}{\mathcal{N}^{k+1}} \alpha^{\frac{k+1}{2}}, & \text { if } 0<k<1 \\ \frac{1}{\mathcal{N}^{2} \varsigma^{1-k}} \alpha, & \text { if } k \geq 1\end{cases}
$$

Proof This lemma is proven similarly [36].

Lemma 5.5 From (5.23), if we can find that $\zeta$ is satisfied, then we have the estimate of $\alpha$ as follows:

$$
\alpha^{-1} \leq \begin{cases}\left(\frac{\mathcal{V}_{3}(\bar{E}, \underline{E}, s)}{\left(\zeta-\sqrt{2}\left(1+\xi_{1}^{2} \bar{D}_{5}^{2}\right)^{\frac{1}{2}}\right)}\right)^{\frac{2}{s+1}}\left(\frac{\mathcal{C}}{\epsilon}\right)^{\frac{2}{s+1},}, & \text { if } 0<s<1, \\ \frac{\mathcal{V}_{4}(\bar{E}, E)}{\left(\zeta-\sqrt{2}\left(1+\xi_{1}^{2} \bar{D}_{5}^{2}\right)^{\frac{1}{2}}\right)}\left(\frac{\mathcal{C}}{\epsilon}\right), & \text { if } s \geq 1 .\end{cases}
$$

Proof From (5.23), it gives

$$
\begin{aligned}
\zeta \epsilon & =\left\|\mathcal{K} \mathcal{P}_{\alpha, \delta}^{\epsilon}-\Xi^{\epsilon}\right\|_{L^{2}\left([0, R] ; r^{2}\right)} \leq\left\|\sum_{j=1}^{+\infty} \frac{\alpha}{\left|\Theta_{\beta, j}^{\xi_{1}, \xi_{2}}(\pi, R, T)\right|^{2}+\alpha}\left\langle\Xi^{\epsilon}, \psi_{j}\right\rangle \psi_{j}\right\|_{L^{2}\left([0, R] ; r^{2}\right)} \\
& \leq \underbrace{\left\|\Xi^{\epsilon}-\Xi\right\|_{L^{2}\left([0, R] ; r^{2}\right)}}_{: \mathcal{X}_{1}}+\underbrace{\left\|\sum_{j=1}^{+\infty} \frac{\alpha}{\left|\Theta_{\beta, j}^{\xi_{1}, \xi_{2}}(\pi, R, T)\right|^{2}+\alpha}\left\langle\Xi, \psi_{j}\right\rangle \psi_{j}\right\|_{L^{2}\left([0, R] ; r^{2}\right)}}_{:=\mathcal{X}_{2}} .
\end{aligned}
$$

We have the estimate $\zeta \epsilon$ through two steps as follows, one by one.

Step 1: Estimate of $\mathcal{X}_{1}$, to do this, we recall $\Xi, \Xi^{\epsilon}$ from expressions (5.2) and (5.4), and we have

$$
\begin{aligned}
\mathcal{X}_{1}^{2} & \leq 2\left\|f^{\epsilon}-f\right\|_{L^{2}\left([0, R] ; r^{2}\right)}^{2}+2 \xi_{1}^{2} \bar{D}_{5}^{2}\left\|G^{\epsilon}-G\right\|_{L^{\infty}\left(0, T ; L^{2}\left([0, T] ; r^{2}\right)\right)}^{2} \\
& \leq 2 \epsilon^{2}\left(1+\xi_{1}^{2} \bar{D}_{5}^{2}\right) .
\end{aligned}
$$

Step 2: Estimate of $\mathcal{X}_{2}$, using again the a priori bound condition of $\Xi$, we obtain

$$
\begin{aligned}
\mathcal{X}_{2} & =\left\|\frac{\alpha\left|\Theta_{\beta, j}^{\xi_{1}, \xi_{2}}(\pi, R, T)\right|\left(j^{2}\right)^{-s}}{\left|\Theta_{\beta, j}^{\xi_{1}, \xi_{2}}(\pi, R, T)\right|^{2}+\alpha} \frac{\left(j^{2}\right)^{s}\left\langle\Xi, \psi_{j}\right\rangle \psi_{j}}{\left|\Theta_{\beta, j}^{\xi_{1}, \xi_{2}}(\pi, R, T)\right|}\right\|_{L^{2}\left([0, R] ; r^{2}\right)} \\
& \leq \sup _{j \geq 1}\left(\frac{\alpha\left(j^{2}\right)^{-s}\left|\Theta_{\beta, j}^{\xi_{1}, \xi_{2}}(\pi, R, T)\right|}{\left|\Theta_{\beta, j}^{\xi_{1}, \xi_{2}}(\pi, R, T)\right|^{2}+\alpha}\right) \mathcal{C} .
\end{aligned}
$$

From (5.28), using Lemma 2.4 and Lemma 5.4 implies that

$$
\frac{\alpha\left(j^{2}\right)^{-s} \frac{\bar{E}}{j^{2}}}{\frac{E^{2}}{\bar{j}^{4}}+\alpha} \leq \bar{E} \frac{\alpha\left(j^{2}\right)^{-s-1}}{\underline{E}^{2}+\alpha j^{4}} \leq \begin{cases}\underbrace{\bar{E} \frac{(s+1)^{s+1}}{2 E^{s+1}}}_{:=\mathcal{V}_{1}(\bar{E}, \underline{E}, s)} \alpha^{\frac{s+1}{2}}, & \text { if } 0<s<1, \\ \underbrace{\frac{\bar{E}}{E^{2}}}_{\mathcal{V}_{2}(\bar{E}, \underline{E})} \alpha, & \text { if } s \geq 1 .\end{cases}
$$


From the analytics assessment on the side, we get

$$
\epsilon\left(\zeta-\sqrt{2}\left(1+\xi_{1}^{2} \bar{D}_{5}^{2}\right)^{\frac{1}{2}}\right) \leq \begin{cases}\mathcal{V}_{3}(\bar{E}, \underline{E}, s) \mathcal{C} \alpha^{\frac{s+1}{2}}, & \text { if } 0<s<1 \\ \mathcal{V}_{4}(\bar{E}, \underline{E}) \mathcal{C} \alpha, & \text { if } s \geq 1\end{cases}
$$

This yields

$$
\alpha^{-1} \leq \begin{cases}\left(\frac{\mathcal{V}_{3}(\bar{E}, \underline{E}, s)}{\left(\zeta-\sqrt{2}\left(1+\xi_{1}^{2} \bar{D}_{5}^{2}\right)^{\frac{1}{2}}\right)}\right)^{\frac{2}{s+1}}\left(\frac{\mathcal{C}}{\epsilon}\right)^{\frac{2}{s+1}}, & \text { if } 0<s<1 \\ \frac{\mathcal{V}_{4}(\bar{E}, \underline{E})}{\left(\zeta-\sqrt{2}\left(1+\xi_{1}^{2} \bar{D}_{5}^{2}\right)^{\frac{1}{2}}\right)}\left(\frac{\mathcal{C}}{\epsilon}\right), & \text { if } s \geq 1\end{cases}
$$

Theorem 5.6 Assume that (1.3) holds, recalling the $\alpha$ in Lemma 5.5, then we have the following estimate:

$$
\begin{aligned}
& \left\|u_{\alpha, \delta}^{\epsilon}(\cdot, 0)-u(\cdot, 0)\right\|_{L^{2}\left([0, R] ; r^{2}\right)} \\
& \leq \underline{E}^{-\frac{s}{s+1}(1+\zeta)^{\frac{s}{s+1}} \epsilon^{\frac{s}{s+1}} \mathcal{C} \frac{1}{s+1}} \\
& \quad+ \begin{cases}2 \mathcal{Z}_{\delta}^{\frac{1}{2}}\left(1+\xi_{1}^{2} \bar{D}_{5}^{2}\right)^{\frac{1}{2}}\left(\frac{\mathcal{V}_{3}(\bar{E}, \underline{E}, s)}{\left(\zeta-\sqrt{2}\left(1+\xi_{1}^{2} \bar{D}_{5}^{2}\right)^{\frac{1}{2}}\right)}\right)^{\frac{1}{s+1}} \mathcal{C} \frac{1}{s+1} \epsilon^{\frac{s}{s+1}}, & \text { if } 0<s<1, \\
2 \mathcal{Z}_{\delta}^{\frac{1}{2}}\left(1+\xi_{1}^{2} \bar{D}_{5}^{2}\right)^{\frac{1}{2}}\left(\frac{\mathcal{V}_{4}(\bar{E}, \underline{E})}{\left(\zeta-\sqrt{2}\left(1+\xi_{1}^{2} \bar{D}_{5}^{2}\right)^{\frac{1}{2}}\right)}\right)^{\frac{1}{2}} \mathcal{C}^{\frac{1}{2}} \epsilon^{\frac{1}{2}}, & \text { if } s \geq 1 .\end{cases}
\end{aligned}
$$

Proof From the triangle inequality, we get

$$
\begin{aligned}
& \left\|u_{\alpha, \delta}^{\epsilon}(\cdot, 0)-u(\cdot, 0)\right\|_{L^{2}\left([0, R] ; r^{2}\right)} \\
& \quad \leq\left\|u_{\alpha, \delta}^{\epsilon}(\cdot, 0)-u_{\alpha, \delta}(\cdot, 0)\right\|_{L^{2}\left([0, R] ; r^{2}\right)}+\left\|u_{\alpha, \delta}(\cdot, 0)-u(\cdot, 0)\right\|_{L^{2}\left([0, R] ; r^{2}\right)} .
\end{aligned}
$$

From (5.14), we obtain

$$
\left\|u_{\alpha, \delta}^{\epsilon}(\cdot, 0)-u_{\alpha, \delta}(\cdot, 0)\right\|_{L^{2}\left([0, R] ; r^{2}\right)} \leq 2 \mathcal{Z}_{\delta}^{\frac{1}{2}} \alpha^{-\frac{1}{2}} \epsilon\left(1+\xi_{1}^{2} \bar{D}_{5}^{2}\right)^{\frac{1}{2}}
$$

Substituting (5.31) into above equation, one has

$$
\begin{aligned}
& \left\|u_{\alpha, \delta}^{\epsilon}(\cdot, 0)-u_{\alpha, \delta}(\cdot, 0)\right\|_{L^{2}\left([0, R] ; r^{2}\right)} \\
& \quad \leq \begin{cases}2 \mathcal{Z}_{\delta}^{\frac{1}{2}}\left(1+\xi_{1}^{2} \bar{D}_{5}^{2}\right)^{\frac{1}{2}}\left(\frac{\mathcal{V}_{3}(\bar{E}, \underline{\underline{E}, s})}{\left(\zeta-\sqrt{2}\left(1+\xi_{1}^{2} \bar{D}_{5}^{2}\right)^{\frac{1}{2}}\right)}\right)^{\frac{1}{s+1}} \mathcal{C}^{\frac{1}{s+1}} \epsilon^{\frac{s}{s+1},}, & \text { if } 0<s<1, \\
2 \mathcal{Z}_{\delta}^{\frac{1}{2}}\left(1+\xi_{1}^{2} \bar{D}_{5}^{2}\right)^{\frac{1}{2}}\left(\frac{\mathcal{V}_{4}(\bar{E}, \underline{E})}{\left(\zeta-\sqrt{2}\left(1+\xi_{1}^{2} \bar{D}_{5}^{2}\right)^{\frac{1}{2}}\right)}\right)^{\frac{1}{2}} \mathcal{C}^{\frac{1}{2}} \epsilon^{\frac{1}{2}}, & \text { if } s \geq 1 .\end{cases}
\end{aligned}
$$

Next, we give the estimate $\left\|u_{\alpha, \delta}(\cdot, 0)-u(\cdot, 0)\right\|_{L^{2}\left([0, R] ; r^{2}\right)}$ as follows:

$$
\begin{aligned}
& \left\|u_{\alpha, \delta}(\cdot, 0)-u(\cdot, 0)\right\|_{L^{2}\left([0, R] ; r^{2}\right)} \\
& \quad \leq\left\|\sum_{j=1}^{+\infty}\left|\Theta_{\beta, j}^{\xi_{1}, \xi_{2}}(\pi, R, T)\right|^{-1} \frac{\alpha\left\langle\Xi, \psi_{j}\right\rangle \psi_{j}}{\left|\Theta_{\beta, j}^{\xi_{1}, \xi_{2}}(\pi, R, T)\right|^{2}+\alpha}\right\|_{L^{2}\left([0, R] ; r^{2}\right)} .
\end{aligned}
$$


From (5.36), applying the Hölder inequality, we get

$$
\begin{aligned}
& \left\|u_{\alpha, \delta}(\cdot, 0)-u(\cdot, 0)\right\|_{L^{2}\left([0, R] ; r^{2}\right)} \\
& \leq \underbrace{\left\|\sum_{j=1}^{+\infty}\left|\Theta_{\beta, j}^{\xi_{1}, \xi_{2}}(\pi, R, T)\right|^{-1} \frac{\alpha\left\langle\Xi, \psi_{j}\right\rangle \psi_{j}}{\left|\Theta_{\beta, j}^{\xi_{1}, \xi_{2}}(\pi, R, T)\right|^{2}+\alpha}\right\|_{L^{2}\left([0, R] ; r^{2}\right)}^{\frac{s}{s+1}}}_{:=\mathcal{I}_{1}} \\
& \quad \times \underbrace{\left\|\sum_{j=1}^{+\infty} \frac{\alpha}{\left|\Theta_{\beta, j}^{\xi_{1}, \xi_{2}}(\pi, R, T)\right|^{2}+\alpha} \frac{\left\langle\Xi, \psi_{j}\right\rangle \psi_{j}}{\left|\Theta_{\beta, j}^{\xi_{1}, \xi_{2}}(\pi, R, T)\right|}\right\|_{L^{2}\left([0, R] ; r^{2}\right)}^{\frac{1}{s+1}}}_{:=\mathcal{I}_{2}} .
\end{aligned}
$$

Because of $\|\Xi\|_{\mathcal{H}^{s}\left([0, R] ; r^{2}\right)} \leq \mathcal{C}$, we obtain

$$
\mathcal{I}_{2} \leq\left\|\sum_{j=1}^{+\infty}\left(j^{2}\right)^{-s} \frac{\left(j^{2}\right)^{s}\left\langle\Xi, \psi_{j}\right\rangle \psi_{j}}{\left|\Theta_{\beta, j}^{\xi_{1}, \xi_{2}}(\pi, R, T)\right|}\right\|^{\frac{1}{s+1}} \leq \sup _{j \geq 1}\left(j^{2}\right)^{-\frac{s}{s+1}} \mathcal{C}^{\frac{1}{s+1}}
$$

Next, using Lemma 2.4, $\mathcal{I}_{1}$ can be bounded as follows:

$$
\begin{aligned}
\mathcal{I}_{1} \leq & \sup _{j \geq 1}\left|\Theta_{\beta, j}^{\xi_{1}, \xi_{2}}(\pi, R, T)\right|^{-\frac{s}{s+1}}\left\|\sum_{j=1}^{+\infty} \frac{\alpha\left\langle\Xi, \psi_{j}\right\rangle \psi_{j}}{\left|\Theta_{\beta, j}^{\xi_{1}, \xi_{2}}(\pi, R, T)\right|^{2}+\alpha}\right\|_{L^{2}\left([0, R] ; r^{2}\right)}^{\frac{s}{s+1}} \\
\leq & \sup _{j \geq 1}\left(j^{2}\right)^{\frac{s}{s+1}} \underline{E}^{-\frac{s}{s+1}}\left(\left\|\Xi-\Xi^{\epsilon}\right\|_{L^{2}\left([0, R] ; r^{2}\right)}\right. \\
& +\left\|\sum_{j=1}^{+\infty} \frac{\alpha}{\left|\Theta_{\beta, j}^{\xi_{1}, \xi_{2}}(\pi, R, T)\right|^{2}+\alpha}\left\langle\Xi^{\epsilon}, \psi_{j}\right\rangle \psi_{j}(\cdot)\right\|_{L^{2}\left([0, R] ; r^{2}\right)}^{\frac{s}{s+1}} \\
\leq & \sup _{j \geq 1}\left(j^{2}\right)^{\frac{s}{s+1}} \underline{E}^{-\frac{s}{s+1}}(\epsilon+\zeta \epsilon)^{\frac{s}{s+1}} .
\end{aligned}
$$

Combining (5.37) to (5.39), we conclude that

$$
\left\|u_{\alpha, \delta}(\cdot, 0)-u(\cdot, 0)\right\|_{L^{2}\left([0, R] ; r^{2}\right)} \leq \underline{E}^{-\frac{s}{s+1}}(1+\zeta)^{\frac{s}{s+1}} \epsilon^{\frac{s}{s+1}} \mathcal{C}^{\frac{1}{s+1}}
$$

From (5.33) and (5.40), we know that

$$
\begin{aligned}
& \left\|u_{\alpha, \delta}^{\epsilon}(\cdot, 0)-u(\cdot, 0)\right\|_{L^{2}\left([0, R] ; r^{2}\right)} \\
& \leq \underline{E}^{-\frac{s}{s+1}(1+\zeta)^{\frac{s}{s+1}} \epsilon^{\frac{s}{s+1}} \mathcal{C}^{\frac{1}{s+1}}} \\
& \quad+ \begin{cases}2 \mathcal{Z}_{\delta}^{\frac{1}{2}}\left(1+\xi_{1}^{2} \bar{D}_{5}^{2}\right)^{\frac{1}{2}}\left(\frac{\mathcal{V}_{3}(\bar{E}, \underline{\underline{E}}, s)}{\left(\zeta-\sqrt{2}\left(1+\xi_{1}^{2} \bar{D}_{5}^{2}\right)^{\frac{1}{2}}\right)}\right)^{\frac{1}{s+1}} \mathcal{C}^{\frac{1}{s+1}} \epsilon^{\frac{s}{s+1}}, & \text { if } 0<s<1, \\
2 \mathcal{Z}_{\delta}^{\frac{1}{2}}\left(1+\xi_{1}^{2} \bar{D}_{5}^{2}\right)^{\frac{1}{2}}\left(\frac{\mathcal{V}_{4}(\bar{E}, \underline{E})}{\left(\zeta-\sqrt{2}\left(1+\xi_{1}^{2} \bar{D}_{5}^{2}\right)^{\frac{1}{2}}\right)}\right)^{\frac{1}{2}} \mathcal{C}^{\frac{1}{2}} \epsilon^{\frac{1}{2}}, & \text { if } s \geq 1 .\end{cases}
\end{aligned}
$$

This ends the proof of this theorem. 


\section{Conclusion}

In this paper, we focus on the spherically symmetric backward time-fractional diffusion equation with the nonlocal integral condition. By using some properties of the MittagLeffler function, we show two results as follows. First of all, we show the properties of the well-posedness and regularity of the mild solution to this problem. Next, we present that our problem is ill-posed. In addition, we construct a regularized solution and present the convergence rate between the regularized and exact solutions by the fractional Tikhonov method under a priori parameter choice rule and under a posteriori parameter choice rule.

Acknowledgements

The authors are thankful to the area editor for giving valuable comments and suggestions.

Funding

No funding.

Availability of data and materials

Not applicable.

Competing interests

The authors declare that they have no competing interests.

Authors' contributions

All authors conceived of the study, participated in its design and coordination, drafted the manuscript, participated in the sequence alignment, and read and approved the final manuscript.

\section{Author details}

${ }^{1}$ Division of Applied Mathematics, Thu Dau Mot University, Thu Dau Mot City, Binh Duong Province, Vietnam. ${ }^{2}$ School of Mathematics, Iran University of Science and Technology, Narmak, Tehran, Iran.

\section{Publisher's Note}

Springer Nature remains neutral with regard to jurisdictional claims in published maps and institutional affiliations.

Received: 31 August 2021 Accepted: 24 September 2021 Published online: 09 October 2021

\section{References}

1. Alsaedi, A., Ahmad, B., Kirane, M.: Maximum principle for certain generalized time and space fractional diffusion equations. Q. Appl. Math. 73(1), 163-175 (2015)

2. Bianchi, D., Buccini, A., Donatelli, M.: Iterated fractional Tikhonov regularization. Inverse Probl. 31(5), 055005 (2015)

3. Sabatelli, L., Keating, S., Dudley, J., Richmond, P.: Waiting time distributions in financial markets. Eur. Phys. J. B 27, 273-275 (2002)

4. Yuste, S.B., Lindenberg, K.: Subdiffusion-limited reactions. Chem. Phys. 284, 169-180 (2002)

5. Liu, J.J., Yamamoto, M.: A backward problem for the time-fractional diffusion equation. Appl. Anal. 89(11), 1769-1788 (2010)

6. Hall, M.G., Barrick, T.R.: From diffusion-weighted MRI to anomalous diffusion imaging. Magn. Reson. Med. 59, 447-455 (2008)

7. Lazreg, J.E., Abbas, S., Benchohra, M., Karapınar, E.: Impulsive Caputo-Fabrizio fractional differential equations in b-metric spaces. Open Math. 19, 363-372 (2021). https://doi.org/10.1515/math-2021-0040

8. Adiguzel, R.S., Aksoy, U., Karapınar, E., Erhan, I.M.: On the solutions of fractional differential equations via Geraghty type hybrid contractions. Appl. Comput. Math. 20(2), 313-333 (2021)

9. Adiguzel, R.S., Aksoy, U., Karapınar, E., Erhan, I.M.: On the solution of a boundary value problem associated with a fractional differential equation. Math. Methods Appl. Sci. (2021). https://doi.org/10.1002/mma.6652

10. Adiguzel, R.S., Aksoy, U., Karapınar, E., Erhan, I.M.: Uniqueness of solution for higher-order nonlinear fractional differential equations with multi-point and integral boundary conditions. Rev. R. Acad. Cienc. Exactas Fís. Nat., Ser. A Mat. 115, 155 (2021). https://doi.org/10.1007/s13398-021-01095-3

11. Nghia, B.D., Luc, N.H., Binh, H.D., Long, L.D.: Regularization method for the problem of determining the source function using integral conditions. Adv. Theory Nonlinear Anal. Appl. 5(3), 351-362 (2021)

12. Abdelouaheb, A.: Asymptotic stability in Caputo-Hadamard fractional dynamic equations. Res. Nonlinear Anal. 4(2), 77-86 (2021)

13. Podlubny, I.: Fractional Differential Equations. Mathematics in Science and Engineering, vol. 198. Academic Press, San Diego (1990)

14. Cheng, W., Ma, Y.-J., Fu, C.L.: A regularization method for solving the radially symmetric backward heat conduction problem. Appl. Math. Lett. 30, 38-43 (2014)

15. Cheng, W., Fu, C., Qin, F.: Regularization and error estimate for a spherically symmetric backward heat equation. J. Inverse III-Posed Probl. 19(3), 369-377 (2011)

16. Cheng, W., Fu, C.L.: Identifying an unknown source term in a spherically symmetric parabolic equation. Appl. Math. Lett. 26, 387-391 (2013) 
17. Yang, F., Wang, N., Li, X.-X., Huang, C.-Y.: A quasi-boundary regularization method for identifying the initial value of time-fractional diffusion equation on spherically symmetric domain. J. Inverse IIl-Posed Probl. 27(5), 609-621 (2019). https://doi.org/10.1515/jiip-2018-0050

18. Yang, F., Zhang, M., Li, X.-X., Ren, Y.-P.: A posteriori truncated regularization method for identifying unknown heat source on a spherical symmetric domain. Adv. Differ. Equ. 2017, 263 (2017) https://doi.org/10.1186/s13662-017-1276-1

19. Yang, F., Pu, Q., Li, X.-X.: The fractional Tikhonov regularization methods for identifying the initial value problem for a time-fractional diffusion equation. J. Comput. Appl. Math. 380, 112998 (2020). https://doi.org/10.1016/j.cam.2020.112998

20. Shuping, Y., Xiangtuan, X., Yan, N.: Iterated fractional Tikhonov regularization method for solving the spherically symmetric backward time-fractional diffusion equation. Appl. Numer. Math. 160, 217-241 (2021)

21. Alqahtani, B., Aydi, H., Karapınar, E., Rakocevic, V:: A solution for Voltera fractional integral equations by hybrid contractions. Mathematics 7, 694 (2019)

22. Afshari, H., Kalantari, S., Karapınar, E.: Solution of fractional differential equations via couple fixed point. Electron. J. Differ. Equ. 2015, 286 (2015)

23. Salim, A., Benchohra, B., Karapınar, E., Lazreg, J.E.: Existence and Ulam stability for impulsive generalized Hilfer-type fractional differential equations. Adv. Differ. Equ. 2020, 601 (2020)

24. Karapınar, E., Fulga, A., Rashid, M., Shahid, L., Aydi, H.: Large contractions on quasi-metric space with an application to nonlinear fractional differential-equations. Mathematics 2019(7), 444 (2019)

25. Tuan, N.H., Baleanu, D., Thach, T.N., O'Regan, D., Can, N.H.: Approximate solution for a 2-D fractional differential equation with discrete random noise. Chaos Solitons Fractals 133, 109650 (2020)

26. Tuan, N.H., Zhou, Y., Can, N.H.: Identifying inverse source for fractional diffusion equation with Riemann-Liouville derivative. Comput. Appl. Math. 39(2), 1-16 (2020)

27. Phuong, N.D., Hoan, L.V.C., Karapınar, E., Singh, J., Binh, H.D., Can, N.H.: Fractional order continuity of a time semi-linear fractional diffusion-wave system. Alex. Eng. J. 59(6), 4959-4968 (2020)

28. Tuan, N.H., Au, V.V., Can, N.H.: Regularization of initial inverse problem for strongly damped wave equation. Appl. Anal. 97(1), 69-88 (2018)

29. Dokuchaev, N.: On recovering parabolic diffusions from their time-averages. Calc. Var. Partial Differ. Equ. 58(1), Paper No. 27 (2019)

30. Tuan, N.H., Triet, N.A., Luc, N.H., Phuong, N.D.: On a time fractional diffusion with nonlocal in time conditions. Adv. Differ. Equ. 2021, 204 (2021)

31. Thach, T.N., Can, N.H., Tri, V.V.: Identifying the initial state for a parabolic diffusion from their time averages with fractional derivative. Math. Methods Appl. Sci., 1-16 (2021). https://doi.org/10.1002/mma.7179

32. Luc, N.H., Dumitru, B., Agarwal, R.P., Long, L.D.: Identifying the source function for time fractional diffusion with non-local in time conditions. Comput. Appl. Math. 40, 159 (2021). https://doi.org/10.1007/s40314-021-01538-y

33. Phuong, N.D., Baleanu, D., Phong, T.T., Long, L.D.: Recovering the source term for parabolic equation with nonlocal integral condition. Math. Methods Appl. Sci., 1-16 (2021). https://doi.org/10.1002/mma.7331

34. Kirsch, A.: An Introduction to the Mathematical Theory of Inverse Problem. Springer, Berlin (1996)

35. Morigi, S., Reichel, L., Sgallari, F.: Fractional Tikhonov regularization with a nonlinear penalty term. J. Comput. Appl. Math. 324, 142-154 (2017)

36. Tuan, N.H., Huynh, L.N., Baleanu, D., Can, N.H.: On a terminal value problem for a generalization of the fractional diffusion equation with hyper-Bessel operator. Math. Methods Appl. Sci. (2019). https://doi.org/10.1002/mma.6087

37. Engl, H.W., Hanke, M., Neubauer, A.: Regularization of Inverse Problems, vol. 375. Kluwer Academic, Dordrecht (1996)

\section{Submit your manuscript to a SpringerOpen ${ }^{\circ}$ journal and benefit from:}

- Convenient online submission

- Rigorous peer review

- Open access: articles freely available online

- High visibility within the field

- Retaining the copyright to your article

Submit your next manuscript at $\gg$ springeropen.com 